\title{
PARTITION IDENTITIES AND QUIVER REPRESENTATIONS
}

\author{
RICHÁRD RIMÁNYI, ANNA WEIGANDT, AND ALEXANDER YONG
}

\begin{abstract}
We present a particular connection between classical partition combinatorics and the theory of quiver representations. Specifically, we give a bijective proof of an analogue of A. L. Cauchy's Durfee square identity to multipartitions. We then use this result to give a new proof of M. Reineke's identity in the case of quivers $Q$ of Dynkin type $A$ of arbitrary orientation. Our identity is stated in terms of the lacing diagrams of S. AbeasisA. Del Fra, which parameterize orbits of the representation space of $Q$ for a fixed dimension vector.
\end{abstract}

\section{CONTENTS}

1. Introduction 1

1.1. Lace and (multi)partition combinatorics 1

1.2. Quiver Representations 5

2. Proof of Theorem $1.1 \quad 8$

3. Proof of Theorem $1.7 \quad 15$

Acknowledgements 25

References

\section{INTRODUCTION}

The main goal of this paper is to establish a specific connection between classical partition combinatorics and the theory of quiver representations.

1.1. Lace and (multi)partition combinatorics. A lacing diagram [ADF80] $\mathcal{L}$ is a graph. The vertices are arranged in $n$ columns labeled $1,2, \ldots, n$ (left to right). The edges between adjacent columns form a partial matching. A strand is a connected component of $\mathcal{L}$.
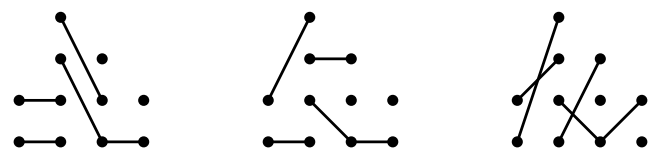

Two lacing diagrams are equivalent if they only differ by reordering of vertices within columns. For example, the lacing diagrams pictured above are all equivalent. Let $\eta=[\mathcal{L}]$ denote the equivalence class of lacing diagrams.

Date: August 5, 2016. 
Pick any $\mathcal{L} \in \eta$ and let $\mathbf{d}(k)$ be the number of vertices in the $k$ th column of $\mathcal{L}$. Define

$$
\operatorname{dim}(\eta):=(\mathbf{d}(1), \ldots, \mathbf{d}(n)) .
$$

Let

$$
\left.s_{i}^{k}(\eta)=\# \text { strands from column } i \text { to column } k-1\right\} \text {, and }
$$$$
t_{j}^{k}(\eta)=\#\{\text { strands starting at column } j \text { using a vertex of column } k\} \text {. }
$$

Fix permutations $\mathbf{w}=\left(w^{(1)}, \ldots, w^{(n)}\right)$, where $w^{(i)} \in \mathfrak{S}_{i}$ and $w^{(i)}(i)=i$. The partition combinatorics behind Theorem 1.1 below suggests the Durfee statistic:

$$
r_{\mathbf{w}}(\eta)=\sum_{k=2}^{n} \sum_{1 \leq i<j \leq k} s_{w^{(k)}(i)}^{k}(\eta) t_{w^{(k)}(j)}^{k}(\eta) .
$$

We will later attach geometric meaning to $r_{\mathbf{w}}(\eta)$ (see Theorem 1.7).

Let

$$
(q)_{k}=(1-q)\left(1-q^{2}\right) \ldots\left(1-q^{k}\right) .
$$

L. Euler introduced the following identity of generating series:

$$
\frac{1}{(q)_{k}}=\sum_{r=0}^{\infty} p_{r, k} q^{r}
$$

where $p_{r, k}$ is the number of integer partitions $\lambda=\left(\lambda_{1} \geq \lambda_{2} \geq \cdots \geq \lambda_{\ell(\lambda)}>0\right)$ of size $|\lambda|:=\sum \lambda_{i}$ equal to $r$ and parts of size at most $k$. Therefore it follows that

$$
\prod_{k=1}^{n} \frac{1}{(q)_{\mathbf{d}(k)}}=\sum_{r=0}^{\infty} p_{r, \mathbf{d}} q^{r}
$$

where $p_{r, \mathbf{d}}$ is the number of sequences of multipartitions $\left(\lambda^{(1)}, \ldots, \lambda^{(n)}\right)$ where

$$
\sum_{i=1}^{n}\left|\lambda^{(i)}\right|=r
$$

and $\lambda^{(i)}$ has parts of size at most $\mathbf{d}(i)$.

Theorem 1.1 (Quiver Durfee Identity).

$$
\prod_{k=1}^{n} \frac{1}{(q)_{\mathbf{d}(k)}}=\sum_{\eta} q^{r_{\mathbf{w}}(\eta)} \prod_{k=1}^{n} \frac{1}{(q)_{t_{k}^{k}(\eta)}} \prod_{i=1}^{k-1}\left[\begin{array}{c}
t_{i}^{k}(\eta)+s_{i}^{k}(\eta) \\
s_{i}^{k}(\eta)
\end{array}\right]_{q},
$$

where the sum is taken over $\eta$ such that $\operatorname{dim}(\eta)=(\mathbf{d}(1), \ldots, \mathbf{d}(n))$.

Here

$$
\left[\begin{array}{l}
k \\
j
\end{array}\right]_{q}=\frac{[k]_{q} !}{[j]_{q} ![k-j]_{q} !}=\frac{(q)_{k}}{(q)_{j}(q)_{k-j}}
$$

is the Gaussian binomial coefficient, where $[i]_{q}:=1+q+q^{2}+\cdots+q^{i-1}$. In fact, $\left[\begin{array}{l}k \\ j\end{array}\right]_{q}$ is the generating series for partitions whose associated Ferrers shape is contained in a $j \times(k-j)$ rectangle. That is

$$
\left[\begin{array}{l}
k \\
j
\end{array}\right]_{q}=\sum_{\lambda \subseteq j \times(k-j)} q^{|\lambda|}
$$


Example 1.2 (Relationship to classical Durfee square identity). Let $n=2$ and set $\mathbf{d}(1)=$ $\mathbf{d}(2)=k$. Then $w^{(1)}=1$ and $w^{(2)}=12$ (throughout we will express permutations in one line notation) by the assumption $w^{(k)}(k)=k$. Equivalence classes of lacing diagrams are determined by the number of strands which start and end at the first vertex. If there are $j$ such strands, then there are $k-j$ strands connecting the first and second vertex. Then there must be exactly $k-(k-j)=j$ strands starting and ending at the second vertex.

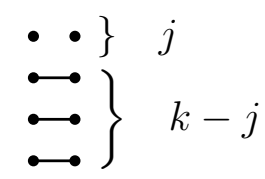

So if $\eta$ has $j$ strands of type $[1,1]$, then

$$
s_{1}^{2}(\eta)=j, \quad t_{1}^{1}(\eta)=j, \quad t_{1}^{2}(\eta)=k-j, \text { and } t_{2}^{2}(\eta)=j .
$$

Thus

Hence (4) states

$$
r_{\mathbf{w}}(\eta)=s_{1}^{2}(\eta) t_{2}^{2}(\eta)=j^{2}
$$

$$
\frac{1}{(q)_{k}} \frac{1}{(q)_{k}}=\sum_{j=0}^{k} q^{j^{2}} \frac{1}{(q)_{k}} \frac{1}{(q)_{j}}\left[\begin{array}{c}
(k-j)+j \\
j
\end{array}\right]_{q} .
$$

Multiplying both sides by $(q)_{k}$ gives the "Durfee square identity" due to A-L. Cauchy:

$$
\frac{1}{(q)_{k}}=\sum_{j=0}^{k} q^{j^{2}}\left[\begin{array}{l}
k \\
j
\end{array}\right]_{q} \frac{1}{(q)_{j}} .
$$

The Durfee square $D(\lambda)$ of $\lambda$ is the largest $j \times j$ square that fits inside $\lambda$. Let $\mathcal{P}_{k}$ be the set of partitions of width at most $k$. By decomposing $\lambda$ using $D(\lambda)$ one obtains a bijection $\mathcal{P}_{k} \stackrel{\sim}{\rightarrow} \bigcup_{j \geq 0} \mathcal{D} \times \mathcal{A}_{j} \times \mathcal{P}_{j}$ where $\mathcal{D}$ is the singleton set consisting of the $j \times j$ square and $\mathcal{A}_{j}$ is the set of partitions contained in a $j \times(k-j)$ rectangle. This gives a textbook bijective proof of (5).

There has been earlier work generalizing the Durfee square identity to multipartitions. In particular, we point the reader to the definition of Durfee dissections of A. Schilling [SW98], which has some similarities in shape to the identity of Theorem 1.1. Here, each Durfee rectangle has at least as many columns as rows, which differs from our definition. We also note the resemblance to the Durfee systems of P. Bouwknegt [Bou02]. Also see the references to loc. cit. for other work on generalized Durfee square identities. One main point of difference is that these identities do not concern lacing diagrams.

Example 1.3. Let $n=3$ and $\mathbf{d}=(1,2,1)$ and $\mathbf{w}=(1,12,123)$. Then

$$
r_{\mathbf{w}}=\left(s_{1}^{2} t_{2}^{2}\right)+\left(s_{1}^{3} t_{2}^{3}+s_{1}^{3} t_{3}^{3}+s_{2}^{3} t_{3}^{3}\right)
$$

and

$$
\prod_{k=1}^{3} \frac{1}{(q)_{t_{k}^{k}}} \prod_{i=1}^{k-1}\left[\begin{array}{c}
t_{i}^{k}+s_{i}^{k} \\
s_{i}^{k}
\end{array}\right]_{q}=\left(\frac{1}{(q)_{t_{1}^{1}}}\right)\left(\frac{1}{(q)_{t_{2}^{2}}}\left[\begin{array}{c}
t_{1}^{2}+s_{1}^{2} \\
s_{1}^{2}
\end{array}\right]_{q}\right)\left(\frac{1}{(q)_{t_{3}^{3}}}\left[\begin{array}{c}
t_{1}^{3}+s_{1}^{3} \\
s_{1}^{3}
\end{array}\right]_{q}\left[\begin{array}{c}
t_{2}^{3}+s_{2}^{3} \\
s_{2}^{3}
\end{array}\right]_{q}\right) .
$$

The table below gives the equivalence classes for $\mathbf{d}=(1,2,1)$ and their corresponding terms on the right hand side of (4). 


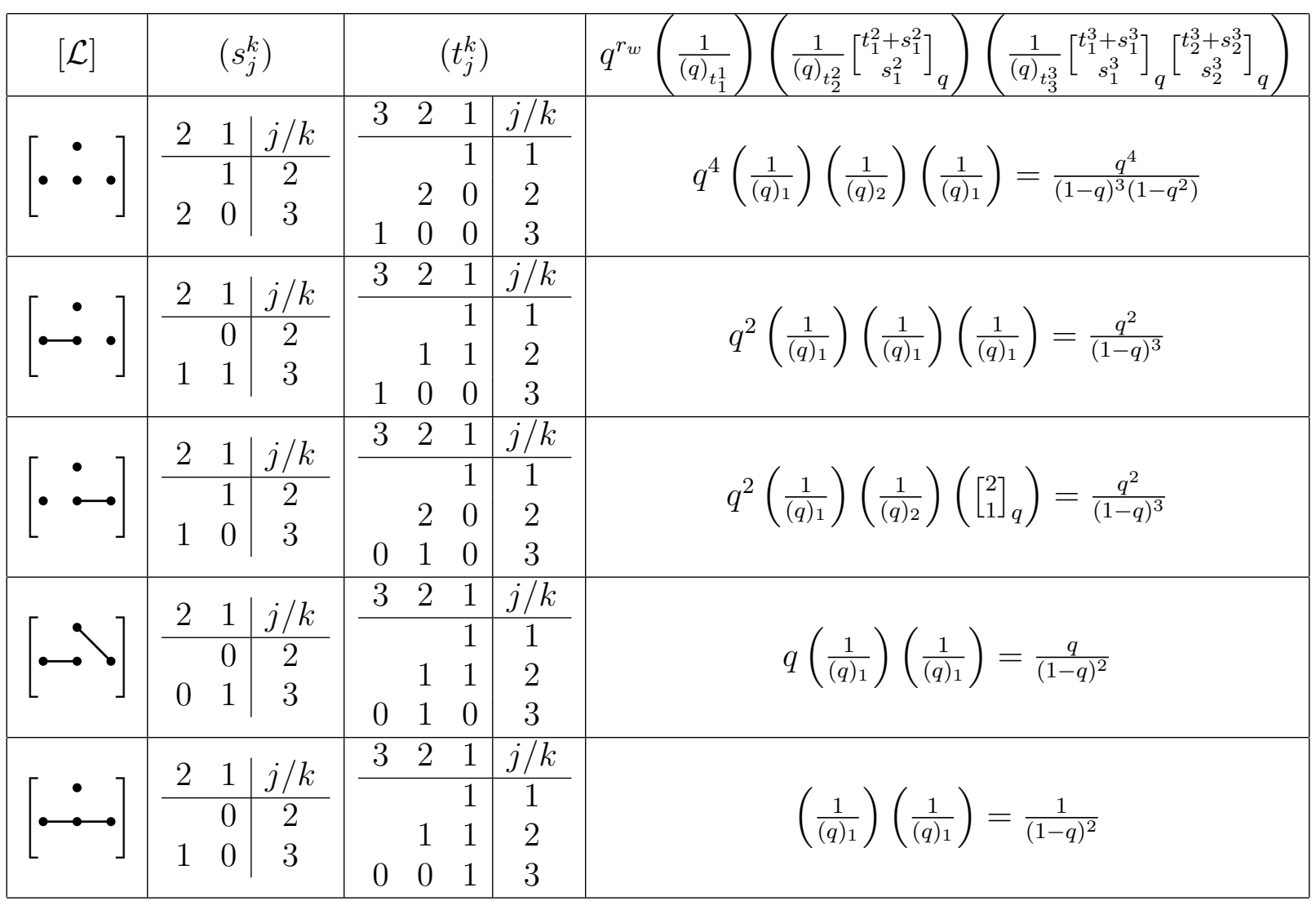

We then verify,

$$
\begin{aligned}
\mathrm{RHS} & =\frac{q^{4}}{(1-q)^{3}\left(1-q^{2}\right)}+\frac{q^{2}}{(1-q)^{3}}+\frac{q^{2}}{(1-q)^{3}}+\frac{q}{(1-q)^{2}}+\frac{1}{(1-q)^{2}} \\
& =\frac{1}{(1-q)^{3}\left(1-q^{2}\right)}\left(q^{4}+q^{2}\left(1-q^{2}\right)+q^{2}\left(1-q^{2}\right)+q(1-q)\left(1-q^{2}\right)+(1-q)\left(1-q^{2}\right)\right) \\
& =\frac{1}{(1-q)^{3}\left(1-q^{2}\right)} \\
& =\frac{1}{(q)_{1}(q)_{2}(q)_{1}} \\
& =\mathrm{LHS} .
\end{aligned}
$$

\section{Notice that (5) says}

and

$$
\frac{1}{(q)_{1}}=1+\frac{q}{(q)_{1}}
$$

$$
\frac{1}{(q)_{2}}=1+\frac{q}{(q)_{1}}\left[\begin{array}{l}
2 \\
1
\end{array}\right]_{q}+\frac{q^{4}}{(q)_{2}}
$$

Thus

$$
\begin{aligned}
\frac{1}{(q)_{1}} \frac{1}{(q)_{2}} \frac{1}{(q)_{1}} & =\left(\frac{1}{(q)_{1}}\right)\left(1+\frac{q}{(q)_{1}}\right)\left(1+\frac{q}{(q)_{1}}\left[\begin{array}{l}
2 \\
1
\end{array}\right]_{q}+\frac{q^{4}}{(q)_{2}}\right) \\
& =\frac{1}{1-q}+\frac{q}{(1-q)^{2}}+\frac{q(1+q)}{(1-q)^{2}}+\frac{q^{2}(1+q)}{(1-q)^{3}}+\frac{q^{4}}{(1-q)^{2}\left(1-q^{2}\right)}+\frac{q^{5}}{(1-q)^{3}\left(1-q^{2}\right)}
\end{aligned}
$$


Theorem 1.1 does not appear to be an a priori consequence of (5). Instead, we will give a bijective proof of Theorem 1.1 in the spirit of the one given for (5) in Example 1.2.

A strand is of type $[i, j]$ if it starts in column $i$ and ends in column $j$. The number of strands of type $[i, j]$ is invariant on $[\mathcal{L}]$. Therefore we let

$$
m_{[i, j]}(\eta)=\#\{\text { strands of type }[i, j] \text { in any } \mathcal{L} \text { of } \eta=[\mathcal{L}]\} .
$$

Corollary 1.4.

$$
\prod_{i=1}^{n} \frac{1}{(q)_{\mathbf{d}(i)}}=\sum_{\eta} q^{r_{\mathbf{w}}(\eta)} \prod_{1 \leq i \leq j \leq n} \frac{1}{(q)_{m_{[i, j]}(\eta)}} .
$$

Proof. From the definitions,

$$
t_{i}^{k}(\eta)+s_{i}^{k}(\eta)=t_{i}^{k-1}(\eta)
$$

Furthermore,

$$
s_{i}^{k}(\eta)=m_{[i, k-1]}(\eta) \text { and } t_{i}^{n}(\eta)=m_{[i, \eta]}(\eta) .
$$

Thus,

$$
\begin{aligned}
\prod_{k=1}^{n} \frac{1}{(q)_{t_{k}^{k}(\eta)}} \prod_{i=1}^{k-1}\left[\begin{array}{c}
\left.t_{i}^{k}(\eta)+s_{i}^{k}(\eta)\right]_{i} \\
s_{i}^{k}(\eta)
\end{array}=\prod_{k=1}^{n} \frac{1}{(q)_{t_{k}^{k}(\eta)}} \prod_{i=1}^{k-1} \frac{(q)_{t_{i}^{k}(\eta)+s_{i}^{k}(\eta)}}{(q)_{t_{i}^{k}(\eta)}(q)_{s_{i}^{k}(\eta)}}\right. \\
=\prod_{k=1}^{n} \frac{1}{(q)_{t_{k}^{k}(\eta)}} \prod_{i=1}^{k-1} \frac{(q)_{t_{i}^{k-1}(\eta)}}{(q)_{t_{i}^{k}(\eta)}(q)_{s_{i}^{k}(\eta)}} \\
=\left(\prod_{k=1}^{n} \frac{1}{(q)_{t_{k}^{k}(\eta)}} \prod_{i=1}^{k-1} \frac{(q)_{t_{i}^{k-1}(\eta)}}{(q)_{t_{i}^{k}}(\eta)}\left(\prod_{k=1}^{n} \prod_{i=1}^{k-1} \frac{1}{(q)_{s_{i}^{k}(\eta)}}\right)\right. \\
=\left(\prod_{k=1}^{n} \prod_{i=1}^{k} \frac{1}{(q)_{t_{i}^{k}(\eta)}}\right)\left(\prod_{k=2}^{n} \prod_{i=1}^{k-1}(q)_{t_{i}^{k-1}(\eta)}\right)\left(\prod_{k=1}^{k-1} \frac{1}{(q)_{s_{i}^{k}(\eta)}}\right) \\
=\left(\prod_{k=1}^{n} \prod_{i=1}^{k} \frac{1}{(q)_{t_{i}^{k}(\eta)}}\right)\left(\prod_{k=1}^{n-1} \prod_{i=1}^{k}(q)_{t_{i}^{k}(\eta)}\right)\left(\prod_{k=1}^{k-1} \frac{1}{(q)_{s_{i}^{k}(\eta)}}\right) \\
=\left(\prod_{i=1}^{n} \frac{1}{(q)_{t_{i}^{n}(\eta)}}\right)\left(\prod_{k=1}^{n} \prod_{i=1}^{k-1} \frac{1}{(q)_{s_{i}^{k}(\eta)}}\right) \\
=\left(\prod_{i=1}^{n} \frac{1}{(q)_{m_{[i, n]}(\eta)}}\right)\left(\prod_{k=1}^{n} \prod_{i=1}^{k-1} \frac{1}{(q)_{m_{[i, k-1]}(\eta)}}\right) \\
=\prod_{1 \leq i \leq j \leq n} \frac{1}{(q)_{m_{[i, j]}}(\eta)} .
\end{aligned}
$$

1.2. Quiver Representations. M. Reineke (cf. [Rim13, (10)]) proved an identity very close to (7) that is the motivation of this work. His identity is phrased in terms of quiver representations; we briefly recall the background essentials. One source concerning quiver representations is [Bri08].

Let $Q$ be a quiver, a directed graph with vertex set $Q_{0}$ and arrows $Q_{1}$. For $a \in Q_{1}$ let $h(a)$ be the head of the arrow and $t(a)$ its tail. Throughout we will work over $\mathbb{C}$. 
A representation $\vee$ of $Q$ assigns a vector space $V_{x}$ to each $x \in Q_{0}$ as well as a linear transformation $V_{a}: V_{t(a)} \rightarrow V_{h(a)}$ for each arrow $a \in Q_{1}$. Each representation $\vee$ of $Q$ has an associated dimension vector

$$
\mathbf{d}: Q_{0} \rightarrow \mathbb{Z}_{\geq 0} \text {, where } \mathbf{d}(x)=\operatorname{dim} V_{x} .
$$

A morphism $\mathrm{T}: \mathrm{V} \rightarrow \mathrm{W}$ is a collection of linear maps $\left(T_{x}: V_{x} \rightarrow W_{x}\right)_{x \in Q_{0}}$ such that

$$
T_{h(a)} V_{a}=W_{a} T_{t(a)} \text { for every arrow } a \in Q_{1} .
$$

Write $\operatorname{Hom}(\mathrm{V}, \mathrm{W})$ for the space of morphisms from $\mathrm{V}$ to $\mathrm{W}$. Given representations $\mathrm{V}$ and $\mathrm{W}$, we may form the direct sum $\mathrm{V} \oplus \mathrm{W}$ by pointwise taking direct sums of vector spaces and morphisms. If $\mathrm{V} \cong \mathrm{V}^{\prime} \oplus \mathrm{V}^{\prime \prime}$ implies $\mathrm{V}^{\prime}$ or $\mathrm{V}^{\prime \prime}$ is trivial, then $\mathrm{V}$ is indecomposable. If $\mathrm{V}$ is a finite dimensional representation of $Q$ then the Krull-Schmidt decomposition is

$$
\mathrm{V} \cong \bigoplus_{i=1}^{m} \mathrm{~V}_{i}^{\oplus m_{i}}
$$

where the $V_{i}$ are pairwise non-isomorphic indecomposable representations. This decomposition and the multiplicities $m_{i}$ are unique up to reordering.

Let Mat $(m, n)$ be the space of $m \times n$ matrices. The representation space is

$$
\operatorname{Rep}_{Q}(\mathbf{d}):=\bigoplus_{a \in Q_{1}} \operatorname{Mat}(\mathbf{d}(h(a)), \mathbf{d}(t(a)) .
$$

$\operatorname{Rep}_{Q}(\mathbf{d})$ is isomorphic to affine space $\mathbb{A}^{N}$ where $N=\sum_{a \in Q_{1}} \mathbf{d}(h(a)) \mathbf{d}(t(a))$. Points of $\operatorname{Rep}_{Q}(\mathbf{d})$ parameterize $\mathbf{d}$ dimensional representations of $Q$. Let

$$
\mathrm{GL}_{Q}(\mathbf{d}):=\prod_{x \in Q_{0}} \mathrm{GL}(\mathbf{d}(x)) .
$$

$\mathrm{GL}_{Q}(\mathbf{d})$ acts on $\operatorname{Rep}_{Q}(\mathbf{d})$ by base change. Orbits of this action are in bijection with isomorphism classes of $\mathbf{d}$ dimensional representations.

For the remainder of the paper, assume $Q$ is a type $A_{n}$ quiver, i.e. the underlying graph of $Q$ is a path with $n$ vertices. Then $\mathrm{GL}_{Q}(\mathbf{d})$ acts on $\operatorname{Rep}_{Q}(\mathbf{d})$ with finitely many orbits. In particular, these orbits are indexed by equivalence classes of $\mathrm{d}$-dimensional lacing diagrams, as follows.

Identify the vertices of $Q$ with the numbers $1, \ldots, n$ from left to right. Let

$$
\Phi^{+}=\{I=[i, j]: 1 \leq i \leq j \leq n\}
$$

be the set of intervals in $Q$. Label the arrows of $Q$ from left to right $a_{1}$ through $a_{n-1}$. In this case, P. Gabriel's theorem states that isomorphism classes of indecomposables biject with elements of $\Phi^{+}$in the following way. Define $\vee_{I}$ with vector spaces

$$
\left(\mathrm{V}_{I}\right)_{k}= \begin{cases}\mathbb{C} & \text { if } k \in I \\ 0 & \text { otherwise }\end{cases}
$$

and morphisms

$$
\left(\mathrm{V}_{I}\right)_{a}= \begin{cases}\operatorname{id}: \mathbb{C} \rightarrow \mathbb{C} & \text { if } h(a), t(a) \in I \\ 0 & \text { otherwise }\end{cases}
$$


Then by (9),

$$
\mathrm{V} \cong \bigoplus_{I \in \Phi^{+}} \mathrm{V}_{I}^{\oplus m_{I}}
$$

where $m_{[i, j]}$ is the multiplicity of $\mathrm{V}_{I}$ in $\mathrm{V}$. We record this data in a lacing diagram $\mathcal{L}$ which has $m_{[i, j]}$ strands starting in column $i$ and ending in column $j$.

Let $\mathbf{d}=\operatorname{dim}(\eta)$. Write

$$
\mathcal{O}_{\eta}:=\mathrm{GL}_{Q}(\mathbf{d}) \cdot V_{\eta} \subset \operatorname{Rep}_{Q}(\mathbf{d})
$$

where

$$
V_{\eta}:=\bigoplus_{I \in \Phi^{+}} \mathrm{V}_{I}^{\oplus m_{I}}
$$

Write $\operatorname{codim}_{\mathbb{C}}(\eta)$ for the (complex) codimension of $\mathcal{O}_{\eta}$ in $\operatorname{Rep}_{Q}(\mathbf{d})$.

Corollary 1.5 (M. Reineke's identity for type $A_{n}$ quivers). For a fixed dimension vector $\mathrm{d}$ :

$$
\prod_{i=1}^{n} \frac{1}{(q)_{\mathbf{d}(i)}}=\sum_{\eta} q^{\operatorname{codim}_{\mathbb{C}} \eta} \prod_{I \in \Phi^{+}} \frac{1}{(q)_{m_{I}(\eta)}},
$$

where the sum is taken over $\eta$ so that $\operatorname{dim}(\eta)=\mathbf{d}$.

M. Reineke's identity holds more generally for all $A D E$ Dynkin types. It should be possible to treat the other cases in a similar manner, although we do not do so here.

Reineke's identities may be naturally phrased as identities among quantum dilogarithm power series in a non-commutative ring. In this language the identities are closely related to cluster algebras (see e.g., work of V. V. Fock-A. B. Goncharov [FG09] and references therein), wall crossing phenomena (see e.g., the paper [DM16] of B. Davison$\mathrm{S}$. Meinhardt as well as the references therein), and Donaldson-Thomas invariants and Cohomological Hall Algebras (see, e.g., the work of M. Kontsevich-Y. Soibelman [KS11]). This paper is intended to be an initial step towards understanding the rich combinatorics encoded by advanced dilogarithm identities, such as B. Keller's identities [Kel11].

We now explain our proof of Corollary 1.5 as a special case of Corollary 1.4 where $\mathrm{w}$ is determined by $Q$. We define permutations $w_{Q}^{(i)} \in \mathfrak{S}_{i}$ as follows. Let $w_{Q}^{(1)}=1$ and $w_{Q}^{(2)}=12$. For $i \geq 3$ let $\iota$ be the natural inclusion from $\mathfrak{S}_{i-1}$ to $\mathfrak{S}_{i}$ and let $w_{0}^{(i-1)}$ denote the longest permutation in $\mathfrak{S}_{i-1}$. Then we set

$$
w_{Q}^{(i)}=\left\{\begin{array}{l}
\iota\left(w_{Q}^{(i-1)}\right) \text { if } a_{i-2} \text { and } a_{i-1} \text { point in the same direction } \\
\iota\left(w_{Q}^{(i-1)} w_{0}^{(i-1)}\right) \text { if } a_{i-2} \text { and } a_{i-1} \text { point in opposite directions. }
\end{array}\right.
$$

Write $\mathbf{w}_{Q}=\left(w_{Q}^{(1)}, \ldots, w_{Q}^{(n)}\right)$.

Example 1.6. Let $Q$ be the quiver pictured below.

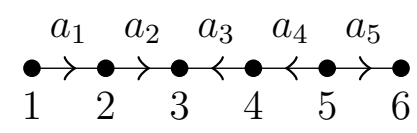

Then $Q$ has associated permutations $\mathbf{w}_{Q}=(1,12,123,3214,32145,541236)$.

With this, it remains to show that the Durfee statistic computes codimension: 


\section{Theorem 1.7.}

$$
r_{\mathbf{w}_{Q}}(\eta)=\operatorname{codim}_{\mathbb{C}}\left(\mathcal{O}_{\eta}\right)
$$

We arrive at Theorem 1.7 by connecting $r_{\mathbf{w}_{Q}}(\eta)$ to an earlier positive combinatorial formula for $\operatorname{codim}_{\mathbb{C}}\left(\mathcal{O}_{\eta}\right)$.

\section{Proof of Theorem 1.1}

Recall the left hand side of (4) is the generating series for an $n$-tuple of partitions, i.e.,

$$
S=\left\{\boldsymbol{\lambda}=\left(\lambda^{(k)}\right)_{1 \leq k \leq n}: \lambda^{(k)} \text { is a partition having parts of size at most } \mathbf{d}(k)\right\}
$$

with respect to the weight:

$$
\mathrm{wt}_{S}(\boldsymbol{\lambda})=\sum_{k=1}^{n}\left|\lambda^{(k)}\right|
$$

Consider the one element set

$$
R(\eta)=\left\{\boldsymbol{\mu}=\left(\mu_{i, j}^{k}\right): \mu_{i, j}^{k} \text { is a } s_{w^{(k)}(i)}^{k}(\eta) \times t_{w^{(k)}(j)}^{k}(\eta) \text { rectangle, } 1 \leq i<j \leq k \leq n\right\},
$$

consisting of a list of rectangles depending on $i, j$, and $k$. Then $r_{\mathbf{w}}(\eta)$ is the total number of boxes in this list of rectangles.

For $i<k$, let $P_{i}^{k}(\eta)$ be the set of partitions which fit inside of an $s_{i}^{k}(\eta) \times t_{i}^{k}(\eta)$ box. Also let $P_{k}^{k}(\eta)$ be the set of partitions which have parts of size at most $t_{k}^{k}(\eta)$. Let

$$
P(\eta)=\left\{\boldsymbol{\nu}=\left(\nu_{i}^{k}\right): \nu_{i}^{k} \in P_{w^{(k)}(i)}^{k}(\eta), 1 \leq i \leq k \leq n\right\} .
$$

Set

Finally, we let

$$
T(\eta)=R(\eta) \times P(\eta)
$$

$$
T=\bigcup_{\eta} T(\eta),
$$

with the union taken over all lace equivalence classes $\eta$ of dimension $\mathbf{d}$.

The right hand side of (4) is the generating series for $T$, with respect to the weight that assigns $(\boldsymbol{\mu}, \boldsymbol{\nu}) \in T$ to

$$
\mathrm{wt}_{T}(\boldsymbol{\mu}, \boldsymbol{\nu})=\sum_{1 \leq i<j<k \leq n}\left|\mu_{i, j}^{k}\right|+\sum_{1 \leq i \leq k \leq n}\left|\nu_{i}^{k}\right| .
$$

Define a map $\Psi: T \rightarrow S$ by "gluing" the partitions of $T$ as indicated in Figure 1, for $1 \leq k \leq n$.

Thus, Theorem 1.1 follows from:

Theorem 2.1. $\Psi: T \rightarrow S$ is a weight-preserving bijection, i.e., $\operatorname{wt}_{T}(\boldsymbol{\mu}, \boldsymbol{\nu})=\mathrm{wt}_{S}(\Psi(\boldsymbol{\mu}, \boldsymbol{\nu}))$.

Proof. $\Psi$ is well-defined: This follows immediately from that fact that if $\operatorname{dim}(\eta)=\mathbf{d}$ then

$$
t_{1}^{k}(\eta)+\ldots+t_{k}^{k}(\eta)=\mathbf{d}(k) .
$$

$\Psi$ is weight-preserving: That $\left.\mathrm{wt}_{T}(\boldsymbol{\mu}, \boldsymbol{\nu})\right)=\mathrm{wt}_{S}(\Psi(\boldsymbol{\mu}, \boldsymbol{\nu}))$ is clear since $\Psi$ preserves the total number of boxes. 


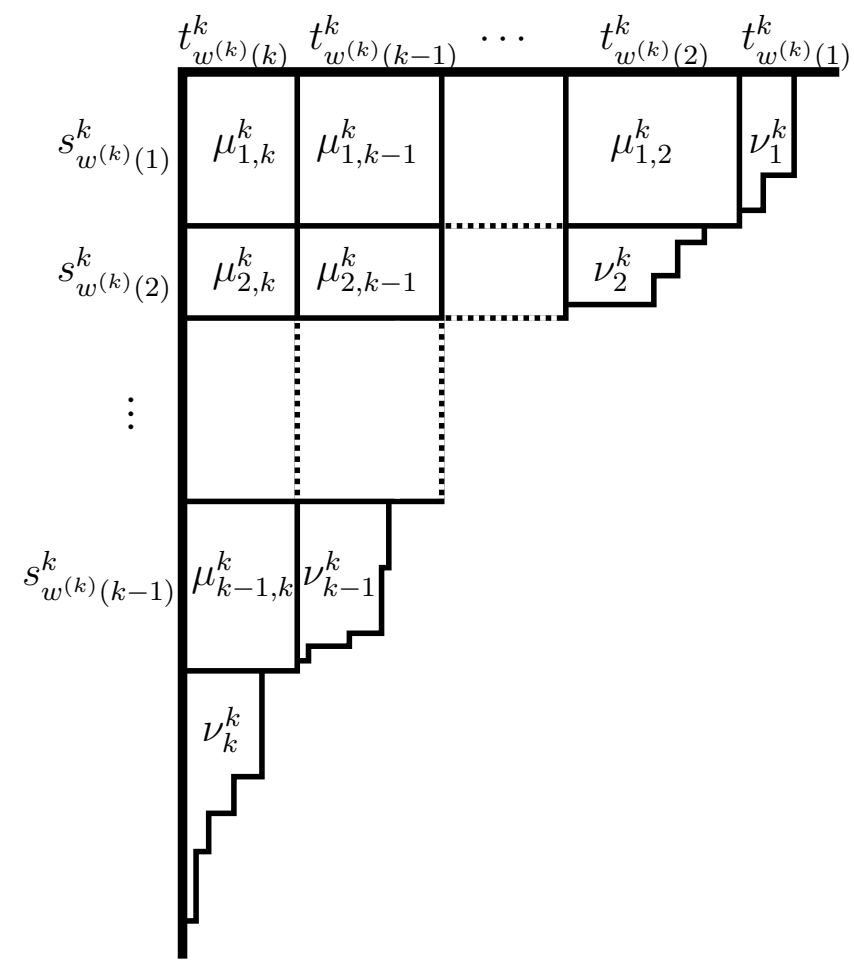

FIGURE 1. Description of the $k$-th component of the map $\Psi: T \rightarrow S$

Definition of $\Phi: S \rightarrow T$ : Given a partition $\lambda$, and $i \in \mathbb{Z}$, the Durfee rectangle $D(\lambda, i)$ is the rectangle with top left corner positioned at $(0,0)$ and bottom right corner where the line $x+y=i$ intersects the (infinite) boundary line of the partition. Equivalently, this is the largest $s \times(s+i)$ rectangle which fits in $\lambda$, justified against the top left corner. (By convention, we define 0 -width and 0-height rectangles as fitting in $\lambda$.)

Example 2.2. Let $\lambda=(3,3,2,2,1)$. Pictured below are the Durfee rectangles $D(\lambda, i)$ for $i=-1,0,4$.
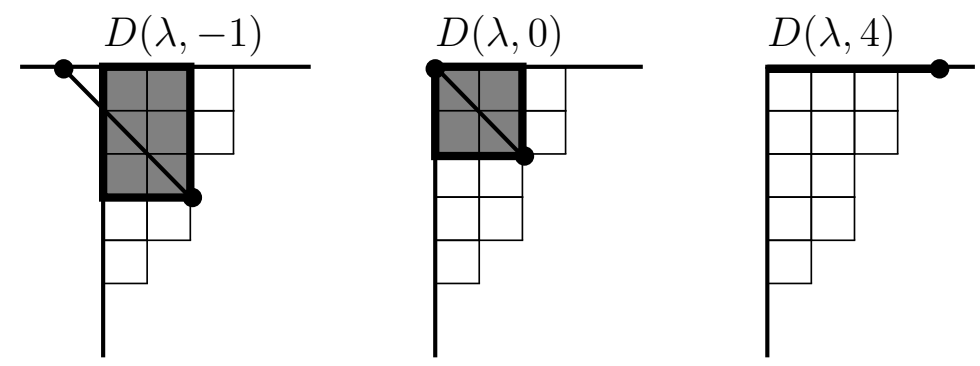

Notice that $D(\lambda, 4)=0 \times 4$ rectangle. The line $x+y=4$ intersects the boundary of $\lambda$ at the point $(4,0)$.

To define $\Phi$, we need to first recursively define parameters $t_{i}^{k}$ for $1 \leq i \leq k$. Our initial condition is that $t_{1}^{1}=d(1)$. Assume $t_{1}^{k-1}, \ldots, t_{k-1}^{k-1}$ has been previously determined. Let

$$
\delta_{i}^{k}=D\left(\lambda^{(k)}, \mathbf{d}(k)-\left(t_{w^{(k)}(1)}^{k-1}+\ldots+t_{w^{(k)}(i)}^{k-1}\right)\right) \text { for } i=1, \ldots, k-1 .
$$




\section{Suppose}

$$
\delta_{i}^{k}=a_{i}^{k} \times b_{i}^{k} \text { rectangle. }
$$

Let

$$
t_{w^{(k)}(i)}^{k}=\mathbf{d}(k)-b_{i}^{k}-\left(t_{w^{(k)}(1)}^{k}+\ldots+t_{w^{(k)}(i-1)}^{k}\right) \text { for } i=1, \ldots, k-1 .
$$

Finally, let

$$
t_{w^{(k)}(k)}^{k}=t_{k}^{k}=\mathbf{d}(k)-\left(t_{w^{(k)}(1)}^{k}+\ldots t_{w^{(k)}(k-1)}^{k}\right) .
$$

Continue this procedure until $k=n$.

Notice that by construction, we have:

Claim 2.3. For $2 \leq k \leq n$,

and

$$
a_{1}^{k} \leq a_{2}^{k} \leq \cdots \leq a_{k-1}^{k}
$$

$$
b_{1}^{k} \geq b_{2}^{k} \geq \cdots \geq b_{k-1}^{k} .
$$

We now also fix parameters $s_{i}^{k}$ for $1 \leq i \leq k-1$. Here we set

$$
s_{w^{(k)}(1)}^{k}=a_{1}^{k}
$$

and

$$
s_{w^{(k)}(i)}^{k}=a_{i}^{k}-a_{i-1}^{k} \text { for } i=2, \ldots, k-1 .
$$

These parameters are nonnegative integers, by Claim 2.3 .

Claim 2.4. $t_{w^{(k)}(i)}^{k}+s_{w^{(k)}(i)}^{k}=t_{w^{(k)}(i)}^{k-1}$ for $1 \leq i<k \leq n$.

Proof. Fix $k$. Our proof is by induction on $i$.

In the base case $i=1$, we have

$$
\begin{aligned}
t_{w^{(k)}(1)}^{k}+s_{w^{(k)}(1)}^{k} & =\mathbf{d}(k)-b_{1}^{k}+a_{1}^{k} \\
& =\mathbf{d}(k)-\left(\mathbf{d}(k)-t_{w^{(k)}(1)}^{k-1}+a_{1}^{k}\right)+a_{1}^{k}
\end{aligned}
$$

Now assume

holds for all $j<i$. Then

$$
t_{w^{(k)}(j)}^{k}+s_{w^{(k)}(j)}^{k}=t_{w^{(k)}(j)}^{k-1}
$$

$$
\begin{aligned}
t_{w^{(k)}(i)}^{k}+s_{w^{(k)}(i)}^{k}= & \mathbf{d}(k)-b_{i}-\left(t_{w^{(k)}(1)}^{k}+\ldots+t_{w^{(k)}(i-1)}^{k}\right)+a_{i}^{k}-a_{i-1}^{k} \\
= & \mathbf{d}(k)-\left(\mathbf{d}(k)-\left(t_{w^{(k)}(1)}^{k-1}+\ldots+t_{w^{(k)}(i)}^{k-1}\right)+a_{i}^{k}\right) \\
& -\left(t_{w^{(k)}(1)}^{k}+\ldots+t_{w^{(k)}(i-1)}^{k}\right)+a_{i}^{k}-a_{i-1}^{k} \\
= & t_{w^{(k)}(i)}^{k-1}+\left(t_{w^{(k)}(1)}^{k-1}-t_{w^{(k)}(1)}^{k}\right)+\ldots \\
& +\left(t_{w^{(k)}(i-1)}^{k-1}-t_{w^{(k)}(i-1)}^{k}\right)-a_{i-1}^{k} \\
= & t_{w^{(k)}(i)}^{k-1}+s_{w^{(k)}(1)}^{k}+\ldots+s_{w^{(k)}(i-1)}^{k}-a_{i-1}^{k} \\
= & t_{w^{(k)}(i)}^{k-1} .
\end{aligned}
$$


Claim 2.5. Let $\eta(\boldsymbol{\lambda})$ be the equivalence class of a lacing diagram uniquely defined by requiring that the number of strands:

- from $i$ to $j$ is $s_{i}^{j+1}$ for $1 \leq i \leq j \leq n-1$;

- from $i$ to $n$ is $t_{i}^{n}$ for $i=1 \ldots n$.

Then:

(1) $s_{i}^{k}(\eta(\boldsymbol{\lambda}))=s_{i}^{k}$

(2) $t_{j}^{k}(\eta(\boldsymbol{\lambda}))=t_{j}^{k}$

(3) $\operatorname{dim}(\eta(\boldsymbol{\lambda}))=\mathbf{d}$.

Proof. (1) By hypothesis.

(2) By Claim 2.4. $t_{i}^{k}=t_{i}^{k+1}+s_{i}^{k+1}$. Iterating, we obtain

$$
\begin{array}{rlr}
t_{i}^{k} & =t_{i}^{k+2}+s_{i}^{k+2}+s_{i}^{k+1} \\
& =\ldots \\
& =t_{i}^{n}+\sum_{\ell=k+1}^{n} s_{i}^{\ell} & \\
& =t_{i}^{n}(\eta(\boldsymbol{\lambda}))+\sum_{\ell=k+1}^{n} s_{i}^{\ell}(\eta(\boldsymbol{\lambda})) \quad \text { (by hypothesis) } \\
& =t_{i}^{k}(\eta(\boldsymbol{\lambda})) .
\end{array}
$$

(3) Let $\widetilde{\mathbf{d}}=\operatorname{dim}(\eta(\boldsymbol{\lambda}))$. By (2), we have

$$
\mathbf{d}(k)=t_{1}^{k}+\ldots+t_{k}^{k}=t_{1}^{k}(\eta(\boldsymbol{\lambda}))+\ldots+t_{k}^{k}(\eta(\boldsymbol{\lambda}))=\widetilde{\mathbf{d}}(k) .
$$

In view of Claim 2.5, we may disassemble each $\lambda^{(k)}$ as in Figure 1 to obtain rectangles of size

$$
s_{w^{(k)}(i)}^{k}(\eta(\boldsymbol{\lambda})) \times t_{w^{(k)}(j)}^{k}(\eta(\boldsymbol{\lambda}))(\text { where } 1 \leq i<j \leq k)
$$

and partitions

$$
\left.\nu_{i}^{k} \in P_{w^{(k)}(i)}^{k}(\eta(\boldsymbol{\lambda})) \text { (where } 1 \leq i \leq k\right) .
$$

That is, we have associated to $\boldsymbol{\lambda}$ a pair $(\boldsymbol{\mu}, \boldsymbol{\nu}) \in T(\eta(\boldsymbol{\lambda})) \subseteq T$. This shows $\Phi: S \rightarrow T$, as desired.

$\Phi$ is weight-preserving: This is clear.

Example 2.6. Let $Q$ be an equioriented quiver on 3 vertices, i.e. all arrows point in the same direction.

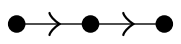

Then $\mathbf{w}_{Q}=(1,12,123)$. Fix a dimension vector $\mathbf{d}=(3,6,5)$ and partitions

$$
\lambda^{(1)}=(2,1), \lambda^{(2)}=(5,1) \text {, and } \lambda^{(3)}=(3,3,2,1,1) .
$$




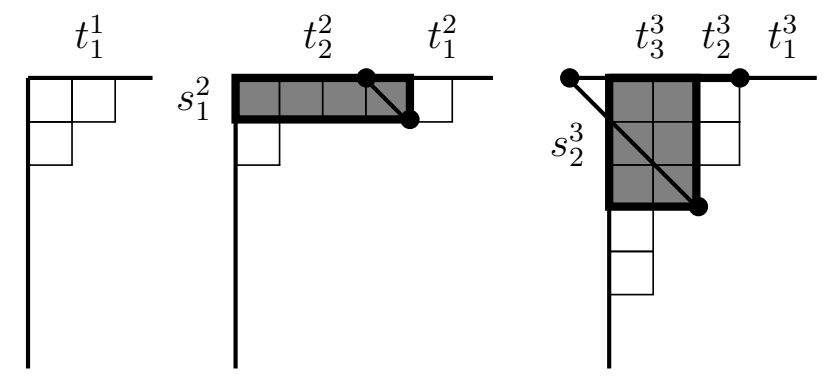

Then

$$
\delta_{1}^{2}=D\left(\lambda^{(2)}, 6-3\right)=1 \times 4 \text { rectangle, } t_{1}^{2}=2 \text {, and } t_{2}^{2}=4 .
$$

From this, we have

$$
\delta_{1}^{3}=D\left(\lambda^{(3)}, 5-2\right)=0 \times 3 \text { and } \delta_{2}^{3}=D\left(\lambda^{(3)}, 5-2-4\right)=3 \times 2 \text { rectangles. }
$$

So $t_{1}^{3}=2, t_{2}^{3}=1$, and $t_{3}^{3}=2$. This corresponds to $\eta(\boldsymbol{\lambda})=[\mathcal{L}]$ where

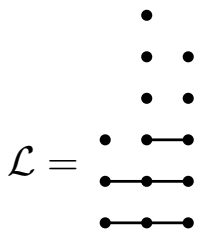

Alternatively, if $Q$ is bipartite, that is adjacent arrows point in opposite directions, then $\mathbf{w}_{Q}=(1,12,213)$.

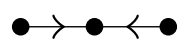

Keeping the same dimension vector and partitions $\lambda^{(k)}$ gives the following.
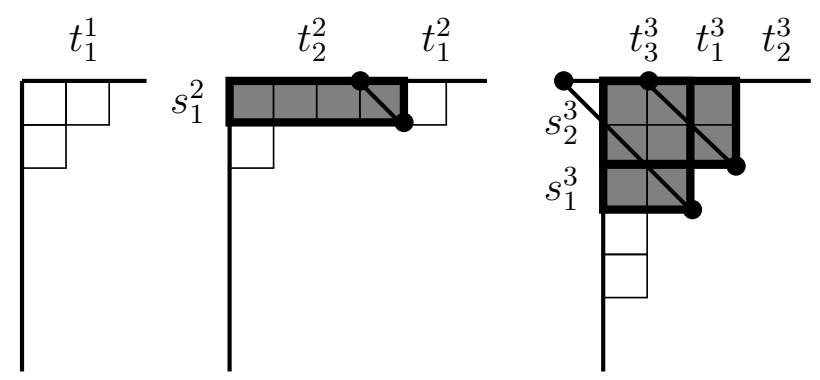

As before,

$$
\delta_{1}^{2}=D\left(\lambda^{(2)}, 6-3\right)=1 \times 4 \text { rectangle }
$$

Consequently,

$$
\delta_{1}^{3}=D\left(\lambda^{(3)}, 5-4\right)=2 \times 3 \text { and } \delta_{2}^{3}=D\left(\lambda^{(3)}, 5-4-2\right)=3 \times 2 \text { rectangles } .
$$

This yields $\eta(\boldsymbol{\lambda})=\left[\mathcal{L}^{\prime}\right]$, where

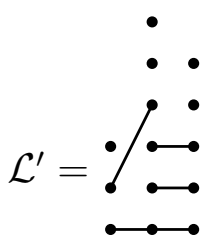


It remains to establish:

Claim 2.7. $\Phi$ and $\Psi$ are mutual inverses.

Proof. Taking $\boldsymbol{\lambda} \in S$, we have $\Psi(\Phi(\boldsymbol{\lambda}))=\boldsymbol{\lambda}$, since $\Phi$ acts by cutting the $\lambda^{(k)}$ 's into various pieces and $\Psi$ glues these shapes together into their original configurations. Now given $(\boldsymbol{\mu}, \boldsymbol{\nu}) \in T(\eta)$, let $\boldsymbol{\lambda}:=\Psi(\boldsymbol{\mu}, \boldsymbol{\nu})$. We must argue $\eta=\eta(\boldsymbol{\lambda})$. If so, $\Phi(\Psi(\boldsymbol{\mu}, \boldsymbol{\nu}))=(\boldsymbol{\mu}, \boldsymbol{\nu})$.

Since $\boldsymbol{\lambda}=\Psi(\boldsymbol{\mu}, \boldsymbol{\nu})$ and $(\boldsymbol{\mu}, \boldsymbol{\nu}) \in T(\eta)$, each $\lambda^{(k)}$ contains a rectangle

$$
\epsilon_{j}^{k}=\left(\sum_{i=1}^{j} s_{w^{(k)}(i)}^{k}(\eta)\right) \times\left(\sum_{i=j+1}^{k} t_{w^{(k)}(i)}^{k}(\eta)\right)
$$

for all $1 \leq j<k$ as in Figure 1 .

By definition, $\operatorname{dim}(\eta)=\mathbf{d}$. Then it follows

$$
\sum_{i=j+1}^{k} t_{w^{(k)}(i)}^{k}(\eta)=\mathbf{d}(k)-\left(\sum_{i=1}^{j} t_{w^{(k)}(i)}^{k}(\eta)\right) .
$$

From the definitions, $t_{i}^{k}(\eta)+s_{i}^{k}(\eta)=t_{i}^{k-1}(\eta)$. So substituting we have

$$
\sum_{i=j+1}^{k} t_{w^{(k)}(i)}^{k}(\eta)=\mathbf{d}(k)-\sum_{i=1}^{j} t_{w^{k}(i)}^{k-1}(\eta)+\sum_{i=1}^{j} s_{w^{(k)}(i)}^{k}(\eta) .
$$

Substitution of (17) into (16) yields

$$
\epsilon_{j}^{k}=s \times\left(s+\mathbf{d}(k)-\sum_{i=1}^{j} t_{w^{k}(i)}^{k-1}(\eta)\right)
$$

contained in $\lambda^{(k)}$ (where $s=\sum_{i=1}^{j} s_{i}^{k}(\eta)$ ). In particular, by construction, the bottom right corner of $\epsilon_{j}^{k}$ intersects the boundary of $\lambda^{(k)}$ (see Figure 1), i.e. $s$ is the maximum value for which $\epsilon_{j}^{k} \subseteq \lambda^{(k)}$. So by the definition of a Durfee rectangle,

$$
\epsilon_{j}^{k}=D\left(\lambda^{(k)}, \mathbf{d}(k)-\sum_{i=1}^{j} t_{w^{k}(i)}^{k-1}(\eta)\right) .
$$

By (10) and Claim 2.5part (2),

$$
\delta_{j}^{k}=D\left(\lambda^{(k)}, \mathbf{d}(k)-\sum_{i=1}^{j} t_{w^{k}(i)}^{k-1}(\eta(\boldsymbol{\lambda}))\right) .
$$

Then if

$$
\left.\sum_{i=1}^{j} t_{w^{k}(i)}^{k-1}(\eta)=\sum_{i=1}^{j} t_{w^{k}(i)}^{k-1}(\eta(\boldsymbol{\lambda}))\right)
$$

it follows that $\delta_{j}^{k}=\epsilon_{j}^{k}$ since both are Durfee rectangles with the same parameter, and are maximal among such rectangles. 
For $k=2$, since $t_{1}^{1}(\eta)=\mathbf{d}(1)=t_{1}^{1}(\eta(\boldsymbol{\lambda}))$, then

$$
\begin{aligned}
\delta_{1}^{2} & =D\left(\lambda^{(2)}, \mathbf{d}(2)-t_{1}^{1}(\eta)\right) \\
& =D\left(\lambda^{(2)}-\mathbf{d}(2)-t_{1}^{1}(\eta(\boldsymbol{\lambda}))\right) \\
& =\epsilon_{1}^{2},
\end{aligned}
$$

so the Durfee rectangles agree. Assume $\delta_{j}^{k-1}=\epsilon_{j}^{k-1}$ for all $1 \leq j<k-1$. Then in particular, $t_{i}^{k-1}(\eta)=t_{i}^{k-1}(\eta(\boldsymbol{\lambda}))$ for all $1 \leq i \leq k-1$. So by (18), $\delta_{j}^{k}=\epsilon_{j}^{k}$.

Therefore, $s_{i}^{k}(\eta)=s_{i}^{k}(\eta(\boldsymbol{\lambda}))$ for all $1 \leq i<k \leq n$ and $t_{i}^{k}(\eta)=t_{i}^{k}(\eta(\boldsymbol{\lambda}))$ for $1 \leq i \leq k \leq n$. Hence $\eta=\eta(\boldsymbol{\lambda})$.

Actually, the proof of Theorem 2.1 implies an enriched form of Theorem 1.1.

Let

$$
(z ; q)_{k}=(1-q z)\left(1-q^{2} z\right) \cdots\left(1-q^{k} z\right) .
$$

Also, for a lace equivalence class $\eta$, let leftstrands ${ }_{\eta}(j)$ be the number of strands that terminate at column $j$ in some (equivalently any) lace diagram $\mathcal{L} \in \eta$. That is,

$$
\text { leftstrands } \mathbf{s}_{\eta}(j)=\sum_{i=1}^{j} s_{i}^{j+1}(\eta)
$$

Corollary 2.8 (of Theorem 2.1).

$$
\prod_{k=1}^{n} \frac{1}{(z ; q)_{\mathbf{d}(k)}}=\sum_{\eta} q^{r_{\mathbf{w}}(\eta)} \prod_{k=1}^{n} z^{\text {1eftstrand } s_{\eta}(k-1)} \frac{1}{(z ; q)_{t_{k}^{k}(\eta)}} \prod_{i=1}^{k-1}\left[\begin{array}{c}
t_{i}^{k}(\eta)+s_{i}^{k}(\eta) \\
s_{i}^{k}(\eta)
\end{array}\right]_{q} .
$$

Proof. The lefthand side of (20) is the generating series for $S$ with respect to the weight that uses $q$ to mark the number of boxes and $z$ to mark length of the partitions involved. Now, suppose $\lambda^{(k)}$ is a partition of $\boldsymbol{\lambda} \in S$ of length $\ell$. Under the indicated decomposition of Figure 1 .

$$
\ell=\ell\left(\nu_{k}^{k}\right)+\sum_{i=1}^{k-1} s_{w^{(k)}(i)}^{k}=\ell\left(\nu_{k}^{k}\right)+\text { leftstrands } \mathbf{s}_{\eta(k-1)},
$$

where the second equality holds by $(19)$ and reordering terms. Here $\ell\left(\nu_{k}^{k}\right)$ is the length of $\nu_{k}^{k}$. The corollary follows immediately from this and Theorem 2.1 combined.

Theorem 1.1 is therefore the $z=1$ case of Corollary 2.8. By analysis as in Example 1.2 . we obtain, in a special case this Durfee square identity:

$$
\frac{1}{(z ; q)_{k}}=\sum_{j=0}^{\infty} z^{j} q^{j^{2}}\left[\begin{array}{l}
k \\
j
\end{array}\right]_{q} \frac{1}{(z ; q)_{j}} .
$$

In addition, following the argument of the Introduction, from Corollary 2.8 one can thereby deduce an enriched form of M. Reineke's identity. 


\section{Proof of TheOrem 1.7}

First we recall some more background on quiver representations. Given $V$ and $W$ an extension of $\mathrm{V}$ by $\mathrm{W}$ is a short exact sequence of morphisms

$$
0 \rightarrow \mathrm{W} \rightarrow \mathrm{E} \rightarrow \mathrm{V} \rightarrow 0 .
$$

Two extensions are equivalent if the following diagram commutes:

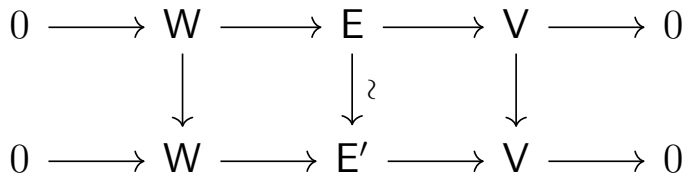

Write $\operatorname{Ext}^{1}(\mathrm{~V}, \mathrm{~W})$ for the space of extensions of $\mathrm{V}$ by $\mathrm{W}$ up to equivalence.

Each quiver has an associated Euler form

$$
\chi_{Q}: \mathbb{N}^{Q_{0}} \times \mathbb{N}^{Q_{0}} \rightarrow \mathbb{Z},
$$

defined by

$$
\chi_{Q}\left(\mathbf{d}_{1}, \mathbf{d}_{2}\right)=\sum_{x \in Q_{0}} \mathbf{d}_{1}(x) \mathbf{d}_{2}(x)-\sum_{a \in Q_{1}} \mathbf{d}_{1}(t(a)) \mathbf{d}_{2}(h(a)) .
$$

Given representations $\mathrm{V}$ and $\mathrm{W}$ of $Q$, use the abbreviation:

$$
\chi_{Q}(\mathrm{~V}, \mathrm{~W}):=\chi_{Q}(\operatorname{dim} \mathrm{V}, \operatorname{dim} W) .
$$

The Euler form relates morphisms and extensions as follows:

$$
\chi_{Q}(\mathrm{~V}, \mathrm{~W})=\operatorname{dimHom}(\mathrm{V}, \mathrm{W})-\operatorname{dimExt}^{1}(\mathrm{~V}, \mathrm{~W}),
$$

(see [Bri08, Corollary 1.4.3]).

Below, we let $a_{x}$ to refer to the arrow of the quiver whose left vertex is $x$. Consider pairs of intervals $(I, J)$ of the following three types:

(I) $I=[w, x-1]$ and $J=[x, z]$ with $w<x \leq z$

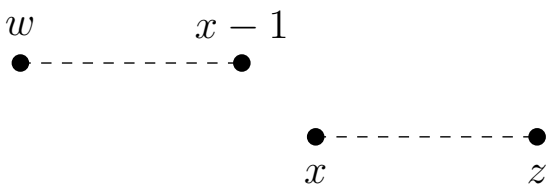

(II) $I=[w, y]$ and $J=[x, z]$ with $w<x \leq y<z$ and the arrows $a_{x-1}$ and $a_{y}$ point in the same direction, e.g.,

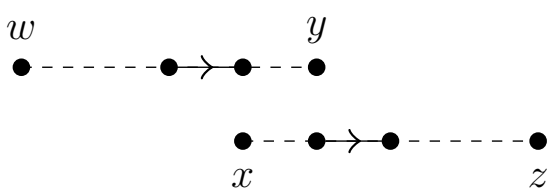

(III) $I=[x, y]$ and $J=[w, z]$ with $w<x \leq y<z$ and the arrows $a_{x-1}$ and $a_{y}$ point in different directions, e.g.,

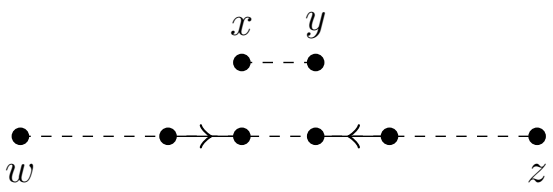


Let

$$
\text { ConditionStrands }=\{(I, J):(I, J) \text { satisfies (I), (II), or (III) }\} .
$$

We also let

$$
\text { StrandPairs }=\left\{(I, J)=\left(\left[x_{1}, x_{2}\right],\left[y_{1}, y_{2}\right]: x_{2} \leq y_{2}\right)\right\} .
$$

(From the definitions (I)-(III), it follows that ConditionStrands $\subset$ StrandPairs.)

Claim 3.1. Fix intervals I and $J$. If $[x, y] \subseteq I, J$ then

$$
\sum_{i=x}^{y} \mathbf{d}_{I}(i) \mathbf{d}_{J}(i)-\sum_{i=x}^{y-1} \mathbf{d}_{I}\left(t\left(a_{i}\right)\right) \mathbf{d}_{J}\left(h\left(a_{i}\right)\right)=1
$$

Proof. Since $[x, y] \subseteq I, J, \mathbf{d}_{I}(i)=\mathbf{d}_{J}(i)=1$ for all $i \in[x, y]$. Therefore,

$$
\sum_{i=x}^{y} \mathbf{d}_{I}(i) \mathbf{d}_{J}(i)=y-x+1 .
$$

Regardless of the orientation of $a_{i}$, if $i \in[x, y-1]$ then $t\left(a_{i}\right), h\left(a_{i}\right) \in[x, y]$. Because $[x, y] \subseteq I, J$, we have $\mathbf{d}_{I}\left(t\left(a_{i}\right)\right)=\mathbf{d}_{J}\left(h\left(a_{i}\right)\right)=1$. So

$$
\sum_{i=x}^{y-1} \mathbf{d}_{I}\left(t\left(a_{i}\right)\right) \mathbf{d}_{J}\left(h\left(a_{i}\right)\right)=(y-1)-x+1 .
$$

Subtracting (25) from (24) gives (23).

Claim 3.2. Let $(I, J) \in$ StrandPairs. Then

$$
(I, J) \in \text { ConditionStrands } \Longleftrightarrow \chi_{Q}\left(\mathrm{~V}_{I}, \mathrm{~V}_{J}\right)<0 \text { or } \chi_{Q}\left(\mathrm{~V}_{J}, \mathrm{~V}_{I}\right)<0 \text {. }
$$

Moreover,

$$
(I, J) \in \text { ConditionStrands } \Rightarrow \chi_{Q}\left(V_{I}, V_{J}\right)=-1 \text { or } \chi\left(V_{J}, V_{I}\right)=-1 \text {. }
$$

Proof. Throughout, given an interval $I$, write $\mathbf{d}_{I}$ for the dimension vector of $\mathrm{V}_{I}$. Applying (21), the definition of the Euler form,

$$
\chi_{Q}\left(\bigvee_{I}, \bigvee_{J}\right)=\chi_{Q}\left(\mathbf{d}_{I}, \mathbf{d}_{J}\right)=\sum_{i=1}^{n} \mathbf{d}_{I}(i) \mathbf{d}_{J}(i)-\sum_{i=1}^{n-1} \mathbf{d}_{I}\left(t\left(a_{i}\right)\right) \mathbf{d}_{J}\left(h\left(a_{i}\right)\right) .
$$

We analyze this expression repeatedly throughout our argument.

$(\Rightarrow)$ By direct computation, we will show if $(I, J) \in$ ConditionStrands then

$$
\chi_{Q}\left(\mathrm{~V}_{I}, \mathrm{~V}_{J}\right)=-1 \text { or } \chi_{Q}\left(\mathrm{~V}_{J}, \mathrm{~V}_{I}\right)=-1,
$$

which is the last assertion of the claim.

Case 1: $(I, J)=([w, x-1],[x, z])$ is of type (I). 
Subcase i: $a_{x-1}$ points to the right.

$$
\begin{aligned}
\chi_{Q}\left(\bigvee_{I}, \bigvee_{J}\right) & =\sum_{i=1}^{n} \mathbf{d}_{I}(i) \mathbf{d}_{J}(i)-\sum_{i=1}^{n-1} \mathbf{d}_{I}\left(t\left(a_{i}\right)\right) \mathbf{d}_{J}\left(h\left(a_{i}\right)\right) \\
& =-\sum_{i=1}^{n-1} \mathbf{d}_{I}\left(t\left(a_{i}\right)\right) \mathbf{d}_{J}\left(h\left(a_{i}\right)\right) \quad(\text { since } I \cap J=\emptyset) \\
& =-\mathbf{d}_{I}\left(t\left(a_{x-1}\right)\right) \mathbf{d}_{J}\left(h\left(a_{x-1}\right)\right) \\
& =-\mathbf{d}_{I}(x-1) \mathbf{d}_{J}(x) \\
& =-1
\end{aligned}
$$

Subcase ii: $a_{x-1}$ points to the left.

Let $Q^{\text {op }}$ be the quiver obtained by reversing the direction of all arrows in $Q$. Then $\chi_{Q}\left(\mathbf{d}_{J}, \mathbf{d}_{I}\right)=\chi_{Q^{\mathrm{op}}}\left(\mathbf{d}_{I}, \mathbf{d}_{J}\right)$. Therefore,

$$
\chi_{Q}\left(\mathrm{~V}_{J}, \bigvee_{I}\right)=\chi_{Q}\left(\mathbf{d}_{J}, \mathbf{d}_{I}\right)=\chi_{Q}^{\mathrm{op}}\left(\mathbf{d}_{I}, \mathbf{d}_{J}\right)=-1
$$

by Subcase 1.i.

Case 2: $(I, J)=([w, y],[x, z])$ is of type (II).

Subcase i: $a_{x-1}$ and $a_{y}$ point to the right.

$$
\begin{aligned}
\chi_{Q}\left(\mathrm{~V}_{I}, \vee_{J}\right)= & \sum_{i=x}^{y} \mathbf{d}_{I}(i) \mathbf{d}_{J}(i)-\sum_{i=x-1}^{y} \mathbf{d}_{I}\left(t\left(a_{i}\right)\right) \mathbf{d}_{J}\left(h\left(a_{i}\right)\right) \\
= & \left(\sum_{i=x}^{y} \mathbf{d}_{I}(i) \mathbf{d}_{J}(i)-\sum_{i=x}^{y-1} \mathbf{d}_{I}\left(t\left(a_{i}\right)\right) \mathbf{d}_{J}\left(h\left(a_{i}\right)\right)\right)-\mathbf{d}_{I}\left(t\left(a_{x-1}\right)\right) \mathbf{d}_{J}\left(h\left(a_{x-1}\right)\right) \\
& -\mathbf{d}_{I}\left(t\left(a_{y}\right)\right) \mathbf{d}_{J}\left(h\left(a_{y}\right)\right) \\
= & 1-\mathbf{d}_{I}\left(t\left(a_{x-1}\right)\right) \mathbf{d}_{J}\left(h\left(a_{x-1}\right)\right)-\mathbf{d}_{I}\left(t\left(a_{y}\right)\right) \mathbf{d}_{J}\left(h\left(a_{y}\right)\right) \quad \text { (Claim 3.1 } \\
= & 1-\mathbf{d}_{I}(x-1) \mathbf{d}_{J}(x)-\mathbf{d}_{I}(y) \mathbf{d}_{J}(y+1) \\
= & -1
\end{aligned}
$$

Subcase ii: $a_{x-1}$ and $a_{y}$ point to the left.

$$
\chi_{Q}\left(\mathrm{~V}_{J}, \mathrm{~V}_{I}\right)=-1 \text { by the } Q^{\mathrm{op}} \text { argument, as in Subcase 1.i. }
$$

Case 3: $(I, J)=([x, y],[y, z])$ is of type (III).

Subcase i: $a_{x-1}$ points right and $a_{y}$ points left.

$$
\begin{aligned}
\chi_{Q}\left(\bigvee_{I}, \bigvee_{J}\right)= & \sum_{i=x}^{y} \mathbf{d}_{I}(i) \mathbf{d}_{J}(i)-\sum_{i=x-1}^{y} \mathbf{d}_{I}\left(t\left(a_{i}\right)\right) \mathbf{d}_{J}\left(h\left(a_{i}\right)\right) \\
= & \left(\sum_{i=x}^{y} \mathbf{d}_{I}(i) \mathbf{d}_{J}(i)-\sum_{i=x}^{y-1} \mathbf{d}_{I}\left(t\left(a_{i}\right)\right) \mathbf{d}_{J}\left(h\left(a_{i}\right)\right)\right)-\mathbf{d}_{I}\left(t\left(a_{x-1}\right)\right) \mathbf{d}_{J}\left(h\left(a_{x-1}\right)\right) \\
& -\mathbf{d}_{I}\left(t\left(a_{y}\right)\right) \mathbf{d}_{J}\left(h\left(a_{y}\right)\right) \\
= & 1-\mathbf{d}_{I}\left(t\left(a_{x-1}\right)\right) \mathbf{d}_{J}\left(h\left(a_{x-1}\right)\right)-\mathbf{d}_{I}\left(t\left(a_{y}\right)\right) \mathbf{d}_{J}\left(h\left(a_{y}\right)\right) \quad \text { (Claim 3.1 } \\
= & 1-\mathbf{d}_{I}(x-1) \mathbf{d}_{J}(x)-\mathbf{d}_{I}(y-1) \mathbf{d}_{J}(y) \\
= & -1
\end{aligned}
$$


Subcase ii: $a_{x-1}$ points left and $a_{y}$ points right.

$\chi_{Q}\left(\mathrm{~V}_{J}, \mathrm{~V}_{I}\right)=-1$ by the $Q^{\mathrm{op}}$ argument, as in Subcase 1.i.

$(\Leftarrow)$ Let $(I, J)=\left(\left[x_{1}, x_{2}\right],\left[y_{1}, y_{2}\right]\right) \in$ StrandPairs and first assume $\chi_{Q}\left(\mathrm{~V}_{I}, \mathrm{~V}_{J}\right)<0$.

Case 1: $I \cap J=\emptyset$. Then $\mathbf{d}_{I}(i)=0$ or $\mathbf{d}_{J}(i)=0$ for all $i \in[1, n]$ and so

$$
\chi_{Q}\left(\mathbf{d}_{I}, \mathbf{d}_{J}\right)=-\sum_{i=1}^{n-1} \mathbf{d}_{I}\left(t\left(a_{i}\right)\right) \mathbf{d}_{J}\left(h\left(a_{i}\right)\right) .
$$

Since $\chi_{Q}\left(\mathbf{d}_{I}, \mathbf{d}_{J}\right)<0$ there must exist an arrow $a_{i}$ with $t\left(a_{i}\right) \in\left[x_{1}, x_{2}\right]$ and $h\left(a_{i}\right) \in\left[y_{1}, y_{2}\right]$. Then $i=x_{2}, a_{i}$ points to the right, and $y_{1}=x_{2}+1$. This implies $(I, J)$ is of type (I).

Case 2: Assume $I \cap J \neq \emptyset$. Since we assume $x_{2} \leq y_{2}$

$$
I \cap J=\left[x_{1}, x_{2}\right] \cap\left[y_{1}, y_{2}\right]=\left[z, x_{2}\right]
$$

where $z \in\left\{x_{1}, y_{1}\right\}$. Then

$$
\begin{aligned}
\chi_{Q}\left(\mathbf{d}_{I}, \mathbf{d}_{J}\right) & =\sum_{i=1}^{n} \mathbf{d}_{I}(i) \mathbf{d}_{J}(i)-\sum_{i=1}^{n-1} \mathbf{d}_{I}\left(t\left(a_{i}\right)\right) \mathbf{d}_{J}\left(h\left(a_{i}\right)\right) \\
& =\sum_{i=z}^{x_{2}} \mathbf{d}_{I}(i) \mathbf{d}_{J}(i)-\sum_{i=z-1}^{x_{2}} \mathbf{d}_{I}\left(t\left(a_{i}\right)\right) \mathbf{d}_{J}\left(h\left(a_{i}\right)\right) \quad \text { (Claim 3.1 } \\
& =1-\mathbf{d}_{I}\left(t\left(a_{z-1}\right)\right) \mathbf{d}_{J}\left(h\left(a_{z-1}\right)\right)-\mathbf{d}_{I}\left(t\left(a_{x_{2}}\right)\right) \mathbf{d}_{J}\left(h\left(a_{x_{2}}\right)\right) .
\end{aligned}
$$

Since $\chi_{Q}\left(\mathbf{d}_{I}, \mathbf{d}_{J}\right)<0$, we must have

$$
\mathbf{d}_{I}\left(t\left(a_{z-1}\right)\right)=\mathbf{d}_{J}\left(h\left(a_{z-1}\right)\right)=\mathbf{d}_{I}\left(t\left(a_{x_{2}}\right)\right)=\mathbf{d}_{J}\left(h\left(a_{x_{2}}\right)\right)=1 .
$$

Therefore,

$$
t\left(a_{z-1}\right), t\left(a_{x_{2}}\right) \in I=\left[x_{1}, x_{2}\right]
$$

and

$$
h\left(a_{z-1}\right), h\left(a_{x_{2}}\right) \in J=\left[y_{1}, y_{2}\right] .
$$

If an arrow $a_{i}$ points to the right, then $h\left(a_{i}\right)=i+1$ and $t\left(a_{i}\right)=i$. If $a_{i}$ points left, $h\left(a_{i}\right)=i$ and $t\left(a_{i}\right)=i+1$. We proceed by analyzing the direction of $a_{x_{2}}$ and $a_{z-1}$. First consider $a_{x_{2}}$. If $a_{x_{2}}$ points left, then $t\left(a_{x_{2}}\right)=x_{2}+1$ and so $x_{2}+1 \in\left[x_{1}, x_{2}\right]$, which is a contradiction. Therefore, we may assume $a_{x_{2}}$ points right.

Now consider the direction of $a_{z-1}$.

If $a_{z-1}$ points to the right, then $t\left(a_{z-1}\right)=z-1 \in\left[x_{1}, x_{2}\right]$ by (26) and so $z>x_{1}$. Since $z \in\left\{x_{1}, y_{1}\right\}$, we must have $z=y_{1}$.

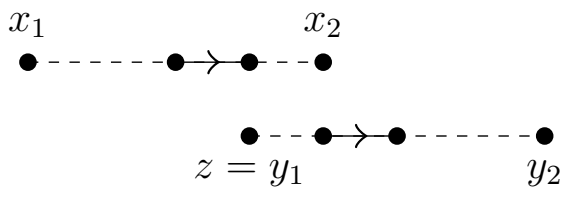

Therefore $(I, J)$ is of type (II).

If $a_{z-1}$ points left, now we have by (27) $h\left(a_{z-1}\right)=z-1 \in\left[y_{1}, y_{2}\right]$. Therefore $z-1>y_{1}$ and so $z \neq y_{1}$ which implies $z=x_{1}$. Hence we have: 


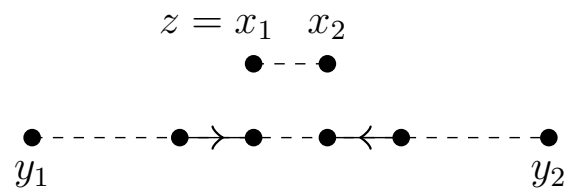

So $(I, J)$ is of type (III).

By near identical arguments, $\chi_{Q}\left(\mathbf{d}_{J}, \mathbf{d}_{I}\right)$ is negative when

(1) $a_{z-1}$ and $a_{x_{2}}$ both point left, $z=y_{1}$, and $x_{2}<y_{2}$; i.e., $(I, J)$ is of type (II)

(2) $a_{z-1}$ points right, $a_{x_{2}}$ points left, $z=x_{1}$ and $x_{2}<y_{2}$ so $(I, J)$ is of type (III).

Proposition 3.3.

$$
\operatorname{codim}_{\mathbb{C}} \eta=\sum_{(I, J) \in \text { Conditionstrands }} m_{I} m_{J}
$$

Proof. There exists a total order on $\Phi^{+}$

$$
\operatorname{Hom}\left(\mathrm{V}_{I}, \mathrm{~V}_{J}\right) \text { and } \operatorname{Ext}^{1}\left(\mathrm{~V}_{J}, \mathrm{~V}_{I}\right)=0 \text { whenever } I<J \text { and } I \neq J,
$$

(see [Rei01], Section 2). Using this ordering and (22), it follows that

$$
\text { if } I<J \text {, then } \chi_{Q}\left(\mathrm{~V}_{I}, \mathrm{~V}_{J}\right) \leq 0 \text { and } \chi_{Q}\left(\mathrm{~V}_{J}, \mathrm{~V}_{I}\right) \geq 0 \text {. }
$$

Voigt's Lemma (see [Rin80, Lemma 2.3]) asserts

$$
\operatorname{codim}_{\mathbb{C}} \eta=\operatorname{dimExt}^{1}\left(\mathrm{~V}_{\eta}, \mathrm{V}_{\eta}\right) \text {. }
$$

Furthermore, indecomposables for Dynkin quivers have no self extensions, that is

$$
\operatorname{Ext}^{1}\left(\mathrm{~V}_{I}, \mathrm{~V}_{I}\right)=0 \text { for all } I \in \Phi^{+} \text {. }
$$

So writing

$$
\mathrm{V}_{\eta} \cong \bigoplus_{I \in \Phi^{+}} \mathrm{V}_{I}^{\oplus m_{I}}
$$

as a finite direct sum of indecomposables, we have

and so

$$
\operatorname{Ext}^{1}\left(\mathrm{~V}_{\eta}, \mathrm{V}_{\eta}\right) \cong \bigoplus_{I<J} \operatorname{Ext}^{1}\left(\mathrm{~V}_{I}, \mathrm{~V}_{J}\right)^{\oplus m_{I} m_{J}}
$$

$$
\operatorname{codim}_{\mathbb{C}} \eta=\sum_{I<J} m_{I} m_{J} \operatorname{dimExt}{ }^{1}\left(\mathrm{~V}_{I}, \mathrm{~V}_{J}\right),
$$

(see [Rim13]). Combining (22) and (28) gives

$$
\operatorname{codim}_{\mathbb{C}} \eta=-\sum_{I<J} m_{I} m_{J} \chi_{Q}\left(\mathrm{~V}_{I}, \mathrm{~V}_{J}\right) .
$$

We will now re-express (30). Let

$$
\begin{gathered}
S=\left\{(I, J): I<J \text { and } \chi_{Q}\left(\mathrm{\vee}_{I}, \mathrm{\vee}_{J}\right)<0\right\}, \\
S_{1}=\left\{(I, J)=\left(\left[x_{1}, x_{2}\right],\left[y_{1}, y_{2}\right]\right):(I, J) \in S \text { and } x_{2} \leq y_{2}\right\}, \text { and } \\
S_{2}=\left\{(I, J)=\left(\left[x_{1}, x_{2}\right],\left[y_{1}, y_{2}\right]\right):(I, J) \in S \text { and } x_{2}>y_{2}\right\} .
\end{gathered}
$$


Trivially, $S=S_{1} \sqcup S_{2}$. Let

$$
\widetilde{S_{2}}=\left\{(J, I):(I, J) \in S_{2}\right\} .
$$

Claim 3.4. ConditionStrands $=S_{1} \sqcup \widetilde{S_{2}}$.

Proof. $S_{1} \cap \widetilde{S_{2}}=\emptyset$, since $(I, J) \in S_{1}$ implies $I<J$ and $(I, J) \in \widetilde{S}_{2}$ implies $I>J$.

$(\subseteq)$ If $(I, J) \in$ ConditionStrands, by Claim 3.2, $\chi_{Q}\left(\mathrm{~V}_{I}, \mathrm{~V}_{J}\right)<0$ or $\chi_{Q}\left(\mathrm{~V}_{J}, \mathrm{~V}_{I}\right)<0$. In the first case, from the definition, $(I, J) \in S_{1}$. In the second case, again by definition, $(J, I) \in S_{2}$, which implies $(I, J) \in \widetilde{S}_{2}$.

$(\supseteq)$ We have $S_{1}, \widetilde{S_{2}} \subseteq$ StrandPairs. Thus by Claim 3.2. $, S_{1}, \widetilde{S_{2}} \subseteq$ ConditionStrands.

Continuing from $(30)$,

$$
\begin{aligned}
\operatorname{codim}_{\mathbb{C}} \eta & =-\sum_{(I, J) \in S} m_{I} m_{J} \chi_{Q}\left(\mathrm{~V}_{I}, \mathrm{~V}_{J}\right) \\
& =-\sum_{(I, J) \in S_{1}} m_{I} m_{J} \chi_{Q}\left(\mathrm{~V}_{I}, \mathrm{~V}_{J}\right)-\sum_{(I, J) \in S_{2}} m_{I} m_{J} \chi_{Q}\left(\mathrm{~V}_{I}, \mathrm{~V}_{J}\right) \\
& =-\sum_{(I, J) \in S_{1}} m_{I} m_{J} \chi_{Q}\left(\mathrm{~V}_{I}, \mathrm{~V}_{J}\right)-\sum_{(I, J) \in \widetilde{S_{2}}} m_{I} m_{J} \chi_{Q}\left(\mathrm{~V}_{J}, \mathrm{~V}_{I}\right) \\
& =\sum_{(I, J) \in S_{1}} m_{I} m_{J}+\sum_{(I, J) \in \widetilde{S_{2}}} m_{I} m_{J}(\text { Claim 3.2) } \\
& =\sum_{(I, J) \in \text { Conditionstrands }} m_{I} m_{J} \quad(\text { Claim 3.4), }
\end{aligned}
$$

as claimed.

Let

$$
\text { BoxStrands } \left.=\left\{\left(\left[w^{(k)}(i), k-1\right],\left[w^{(k)}(j), \ell\right]\right): 1 \leq i<j \leq k \leq \ell \leq n\right)\right\} .
$$

(By definition, if $(I, J)=\left(\left[w^{(k)}(i), k-1\right],\left[w^{(k)}(j), \ell\right] \in\right.$ BoxStrands then $k-1 \leq \ell$, and so $(I, J) \in$ StrandPairs. Thus BoxStrands $\subset$ StrandPairs.)

\section{Proposition 3.5.}

$$
r_{\mathbf{w}}(\eta)=\sum_{(I, J) \in \text { BoxStrands }} m_{I} m_{J}
$$

Proof. By definition (3),

$$
r_{\mathbf{w}}(\eta)=\sum_{k=2}^{n} \sum_{1 \leq i<j \leq k} s_{w^{(k)}(i)}^{k}(\eta) t_{w^{(k)}(j)}^{k}(\eta) .
$$

By definition, $t_{w^{(k)}(j)}^{k}(\eta)$ counts the number of strands in $\eta$ starting at $w^{(k)}(j)$ and using a vertex in column $k$. So

Also,

$$
t_{w^{(k)}(j)}^{k}(\eta)=\sum_{\ell=k}^{n} m_{\left[w^{(k)}(j), \ell\right]}
$$

$$
s_{w^{(k)}(i)}^{k}(\eta)=m_{\left[w^{(k)}(i), k-1\right]} .
$$


Making these substitutions,

$$
\begin{aligned}
r_{\mathbf{w}}(\eta) & =\sum_{k=2}^{n} \sum_{1 \leq i<j \leq k} m_{\left[w^{(k)}(i), k-1\right]}\left(\sum_{\ell=k}^{n} m_{w^{(k)}(j), \ell}\right) \\
& =\sum_{1 \leq i<j \leq k \leq \ell \leq n} m_{\left[w^{(k)}(i), k-1\right]} m_{\left[w^{(k)}(j), \ell\right]} \\
& =\sum_{(I, J) \in \text { BoxStrands }} m_{I} m_{J} .
\end{aligned}
$$

It remains to prove

Lemma 3.6. BoxStrands = ConditionStrands.

Proof. Let $(I, J)$ be as follows:

$$
(I, J):=([x, k-1],[y, \ell]), \text { with } x \neq y, k \leq \ell .
$$

Claim 3.7. All elements of BoxStrands and ConditionStrands may be written in the form (32).

Proof. If

$$
\left(\left[w^{(k)}(i), k-1\right],\left[w^{(k)}(j), \ell\right]\right) \in \text { Boxstrands, }
$$

then

$$
w^{(k)}(i) \neq w^{(k)}(j) \text { and } k \leq \ell .
$$

Hence we are done here by setting $x=w^{(k)}(i)$ and $y=w^{(k)}(j)$.

On the other hand, suppose

$$
\left(\left[x_{1}, x_{2}\right],\left[y_{1}, y_{2}\right]\right) \in \text { ConditionStrands. }
$$

By definition (I)-(III), $x_{1} \neq y_{1}$ and $x_{2}<y_{2}$. So set $x=x_{1}, y=y_{1}, k=x_{2}+1$ and $\ell=y_{2}$.

Claim 3.8. Let $(I, J)$ be as in (32) and suppose $I \cap J=\emptyset$. Then $(I, J) \in$ BoxStrands if and only if $(I, J) \in$ ConditionStrands.

Proof. If $(I, J) \in$ ConditionStrands, then by the disjointness hypothesis it must be of type (I), i.e. of the form

$$
([x, k-1],[k, \ell]) .
$$

Now, since $x \leq k-1$ and as $w^{(k)} \in \mathfrak{S}_{k}$ and $w^{(k)}(k)=k$ there exists $i<k$ such that $w^{(k)}(i)=x$. So

$$
([x, k-1],[k, \ell])=\left(\left[w^{(k)}(i), k-1\right],\left[w^{(k)}(k), \ell\right]\right) \in \text { BoxStrands. }
$$

Conversely, assume

$$
(I, J)=\left(\left[w^{(k)}(i), k-1\right],\left[w^{(k)}(j), \ell\right]\right) \in \text { BoxStrands }
$$

and $I \cap J=\emptyset$. Then $w^{(k)}(j)>k-1$ which means $w^{(k)}(j)=k$ and $j=k$ by the definition of $w^{(k)}$. Furthermore, $w^{(k)}(i) \leq k-1$ since $i<j=k$. So

$$
(I, J)=\left(\left[w^{(k)}(i), k-1\right],[k, \ell]\right) \in \text { ConditionStrands }
$$

is type (I).

Claim 3.9. Let $(I, J)$ be as in (32). Then $(I, J) \in$ BoxStrands $\Leftrightarrow(I, J) \in$ ConditionStrands. 
Proof. We will proceed by induction on $k \geq 2$. In the base case $k=2$, we must have $x=1$ and so $y \geq 2$. As such, $I \cap J=\emptyset$ and so we are done Claim 3.8. Fix $k>2$ and assume the claim holds for $k-1$. That is, given a pair of intervals $\left(\left[x^{\prime}, k-2\right],\left[y^{\prime}, \ell^{\prime}\right]\right)$ so that $x^{\prime}, y^{\prime}$ and $\ell^{\prime}$ satisfy $x^{\prime} \neq y^{\prime}$ and $k-1 \leq \ell^{\prime}$ we have

$$
\left(\left[x^{\prime}, k-2\right],\left[y^{\prime}, \ell^{\prime}\right]\right) \in \text { BoxStrands } \Leftrightarrow\left(\left[x^{\prime}, k-2\right],\left[y^{\prime}, \ell^{\prime}\right]\right) \in \text { ConditionStrands. }
$$

Now let $(I, J)$ be as in (32), i.e.,

$$
(I, J)=([x, k-1],[y, \ell]), \text { with } x \neq y, k \leq \ell .
$$

Again, by Claim 3.8, if $I \cap J=\emptyset$ we are done, so assume $I \cap J \neq \emptyset$. Then $y<k$.

Now, since $1 \leq x, y \leq k$, there exist $i$ and $j$ such that

$$
1 \leq i, j \leq k \text { with } x=w^{(k)}(i) \text { and } y=w^{(k)}(j) .
$$

So from (31)

$$
(I, J)=\left(\left[w^{(k)}(i), k-1\right],\left[w^{(k)}(j), \ell\right]\right) \in \text { BoxStrands } \Longleftrightarrow i<j .
$$

Throughout, when $x \leq k-2$ we write $I^{\prime}:=[x, k-2]$. We will break the argument into two main cases.

Case 1: $a_{k-2}$ and $a_{k-1}$ point in the same direction.

By definition, $w^{(k)}=\iota\left(w^{(k-1)}\right)$. Then if $x \leq k-2$, it follows that

$$
\begin{aligned}
\left(I^{\prime}, J\right) & =([x, k-2],[y, \ell]) \\
& =\left(\left[w^{k-1}(i), k-2\right],\left[w^{k-1}(j), \ell\right]\right)
\end{aligned}
$$

and so

$$
\left(I^{\prime}, J\right) \in \text { BoxStrands if and only if } i<j .
$$

We have four possible subcases, based on the relative values of $x$ and $y$. Subcase i: $x<y=k-1$.

$(I, J)$ is of type (II), and hence $(I, J) \in$ ConditionStrands. Furthermore, note that

$$
\left(I^{\prime}, J\right)=([x, k-2],[k-1, \ell])
$$

is of type (I), and so in ConditionStrands. The intervals for $\left(I^{\prime}, J\right)$ and $(I, J)$ look like this:
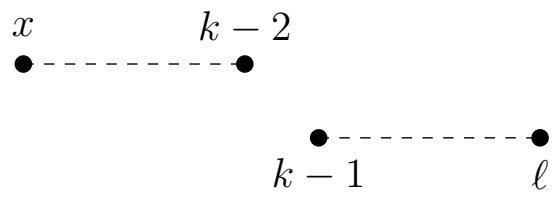

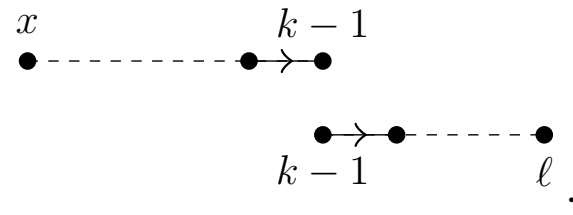

By the inductive hypothesis (33), $\left(I^{\prime}, J\right) \in$ BoxStrands. By (35), $i<j$. Therefore, by (34), $(I, J) \in$ BoxStrands.

Therefore, $(I, J)$ is in both ConditionStrands and BoxStrands.

Subcase ii: $x<y<k-1$. 


$$
\begin{aligned}
(I, J) \in \text { BoxStrands } & \Longleftrightarrow i<j \quad \text { by (34) } \\
& \Longleftrightarrow\left(I^{\prime}, J\right) \in \text { BoxStrands by (35) } \\
& \Longleftrightarrow\left(I^{\prime}, J\right) \in \text { ConditionStrands by (33) } \\
& \Longleftrightarrow a_{x-1} \text { points in the same direction as } a_{k-2} \\
& \Longleftrightarrow a_{x-1} \text { points in the same direction as } a_{k-1} \\
& \Longleftrightarrow(I, J) \in \text { ConditionStrands. }
\end{aligned}
$$

The following picture depicts $\left(I^{\prime}, J\right)$ and $(I, J)$ respectively when $\left(I^{\prime}, J\right)$ and $(I, J)$ are in ConditionStrands.
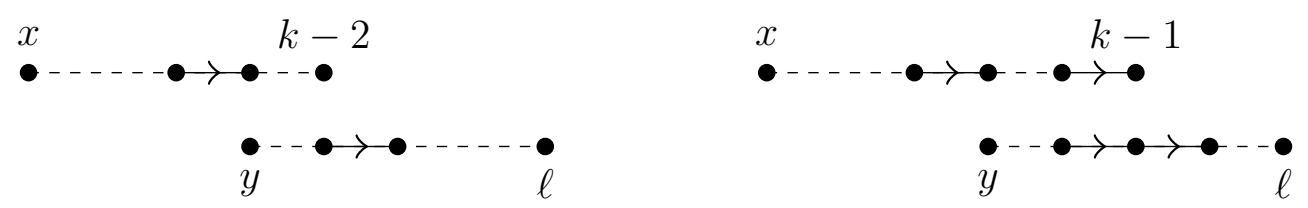

Subcase iii: $y<x=k-1$.

Pictured below are the intervals $I$ and $J$.

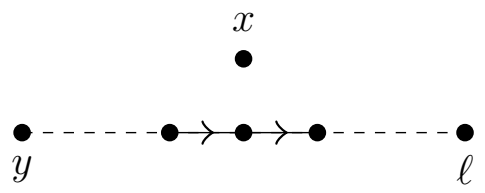

Since $y<x$ and this case assumes $a_{k-2}$ and $a_{k-1}$ point in the same direction, $(I, J)$ cannot be of type (III) and is not in ConditionStrands. Since

$$
w^{(k)}=\iota w^{(k-1)} \text { and } w^{(k-1)}(k-1)=k-1,
$$

it follows that $i=k-1$. Since

$$
y=w^{(k)}(j)=w^{(k-1)}(j)<k-1,
$$

it follows that $i>j$, and so by (34)

$$
(I, J) \notin \text { BoxStrands. }
$$

Therefore, $(I, J)$ is in neither ConditionStrands nor BoxStrands.

Subcase iv: $y<x<k-1$.

$$
\begin{aligned}
(I, J) \in \text { BoxStrands } & \Longleftrightarrow i<j \quad \text { by (34) } \\
& \Longleftrightarrow\left(I^{\prime}, J\right) \in \text { BoxStrands by (35) } \\
& \Longleftrightarrow\left(I^{\prime}, J\right) \in \text { ConditionStrands by (33) } \\
& \Longleftrightarrow a_{x-1} \text { points in the opposite direction as } a_{k-2} \\
& \Longleftrightarrow a_{x-1} \text { points in the opposite direction as } a_{k-1} \\
& \Longleftrightarrow(I, J) \in \text { ConditionStrands. }
\end{aligned}
$$

Below are $\left(I^{\prime} J\right)$ and $(I, J)$ respectively, in the case $\left(I^{\prime}, J\right),(I, J) \in$ ConditionStrands. 

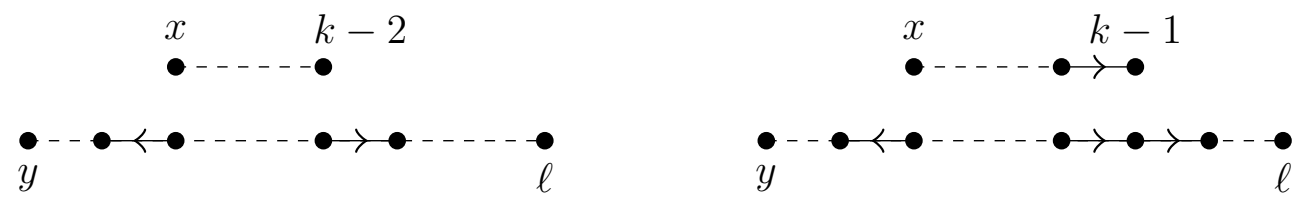

Case 2: $a_{k-2}$ and $a_{k-1}$ point in opposite directions.

By definition,

$$
w^{(k)}=\iota\left(w^{(k-1)} w_{0}^{(k-1)}\right) .
$$

If $x \leq k-2$, and $y \leq k-1$ it follows that

$$
\begin{aligned}
\left(I^{\prime}, J\right) & =([x, k-2],[y, \ell]) \\
& =\left(\left[w^{(k-1)}(k-i), k-2\right],\left[w^{(k-1)}(k-j), \ell\right]\right)
\end{aligned}
$$

and so

$$
\left(I^{\prime}, J\right) \in \text { BoxStrands if and only if } k-i<k-j \text { if and only if } i>j .
$$

Subcase i: $x<y=k-1$.
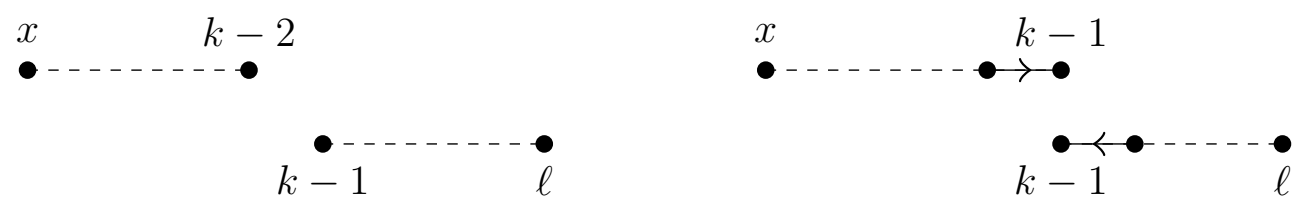

Since $a_{k-2}$ and $a_{k-1}$ point in opposite directions, $(I, J) \notin$ ConditionStrands. The assumption $y=k-1$ implies $\left(I^{\prime}, J\right) \in$ ConditionStrands. By (33) $\left(I^{\prime}, J\right) \in$ BoxStrands. Since $x, y<k$, we have

$$
x=w^{(k)}(i)=w^{(k-1)}(k-i) \text { and } y=w^{(k)}(j)=w^{(k-1)}(k-j) .
$$

Then $k-i<k-j$, so $i>j$ and $(I, J) \notin$ BoxStrands, by (34).

Hence $(I, J)$ is neither in ConditionStrands nor BoxStrands.

Subcase ii: $x<y<k-1$.

$$
\begin{aligned}
(I, J) \in \text { BoxStrands } & \Longleftrightarrow i<j \text { by (34) } \\
& \Longleftrightarrow\left(I^{\prime}, J\right) \notin \text { BoxStrands by (36) } \\
& \Longleftrightarrow\left(I^{\prime}, J\right) \notin \text { ConditionStrands by (33) } \\
& \Longleftrightarrow a_{y-1} \text { points in the opposite direction as } a_{k-2} \\
& \Longleftrightarrow a_{y-1} \text { points in the same direction as } a_{k-1} \\
& \Longleftrightarrow(I, J) \in \text { ConditionStrands. }
\end{aligned}
$$

Below, we have $\left(I^{\prime}, J\right) \notin$ ConditionStrands and $(I, J) \in$ ConditionStrands.
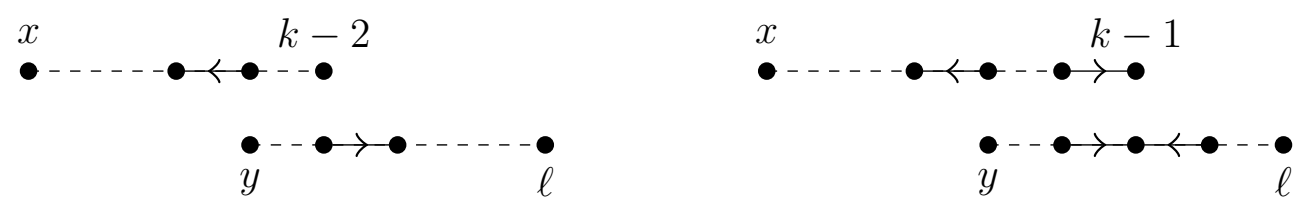

Subcase iii: $y<x=k-1$. Here $(I, J)$ looks like: 


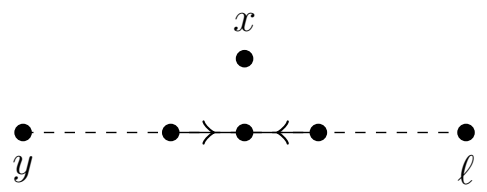

Since Case 2 assumes $a_{k-2}$ and $a_{k-1}$ point in opposite directions, $(I, J)$ is type (II) and so in ConditionStrands. Now,

$$
k-1=x=w^{(k)}(i)=w^{(k-1)}(k-i)
$$

which implies $i=1$. Then $j>i$, so $(I, J) \in$ BoxStrands. So $(I, J)$ is both in ConditionStrands and BoxStrands.

Subcase iv: $y<x<k-1$.

$$
\begin{aligned}
(I, J) \in \text { BoxStrands } & \Longleftrightarrow i<j \text { by }(34) \\
& \Longleftrightarrow\left(I^{\prime}, J\right) \notin \text { BoxStrands by (36) } \\
& \Longleftrightarrow\left(I^{\prime}, J\right) \notin \text { ConditionStrands by (33) } \\
& \Longleftrightarrow a_{x-1} \text { points in the same direction as } a_{k-2} \\
& \Longleftrightarrow a_{x-1} \text { points the opposite direction as } a_{k-1} \\
& \Longleftrightarrow(I, J) \in \text { ConditionStrands. }
\end{aligned}
$$

Pictured below are $\left(I^{\prime}, J\right)$ and $(I, J)$, in the case that $\left(I^{\prime}, J\right) \notin$ ConditionStrands and $(I, J) \in$ ConditionStrands.
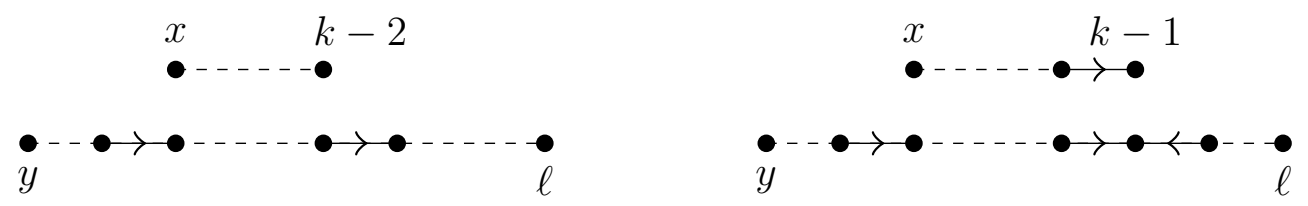

Theorem 1.7 now follows by combining Propositions 3.3 and 3.5 with Lemma 3.6

\section{ACKNOWLEDGEMENTS}

We thank Bruce Berndt and Ae Ja Yee for historical references concerning Durfee square identities. AW and AY were supported by a UIUC Campus Research Board and by an NSF grant.

\section{REFERENCES}

[ADF80] S. Abeasis and A. Del Fra. Degenerations for the representations of an equioriented quiver of type Am, boll. Un. Mat. Ital. Suppl, 2:157-171, 1980.

[Bou02] P. Bouwknegt. Multipartitions, generalized Durfee squares and affine Lie algebra characters. Journal of the Australian Mathematical Society, 72(3):395-408, 2002.

[Bri08] M. Brion. Representations of quivers. 2008.

[DM16] B. Davison and S. Meinhardt. Cohomological Donaldson-Thomas theory of a quiver with potential and quantum enveloping algebras. arXiv:1601.02479, 2016.

[FG09] V. V. Fock and A. B. Goncharov. Cluster ensembles, quantization and the dilogarithm. Ann. Sci. Éc. Norm. Supér. (4), 42(6):865-930, 2009. 
[Kel11] B. Keller. On cluster theory and quantum dilogarithm identities. In Representations of algebras and related topics, EMS Ser. Congr. Rep., pages 85-116. Eur. Math. Soc., Zürich, 2011.

[KS11] M. Kontsevich and Y. Soibelman. Cohomological Hall algebra, exponential Hodge structures and motivic Donaldson-Thomas invariants. Commun. Number Theory Phys., 5(2):231-352, 2011.

[Rei01] M. Reineke. Feigin's map and monomial bases for quantized enveloping algebras. Mathematische Zeitschrift, 237(3):639-667, 2001.

[Rim13] R. Rimányi. On the cohomological Hall algebra of Dynkin quivers. arXiv:1303.3399, 2013.

[Rin80] C. M. Ringel. The rational invariants of the tame quivers. Inventiones mathematicae, 58(3):217-239, 1980.

[SW98] A. Schilling and S. O. Warnaar. Supernomial coefficients, polynomial identities and q-series. The Ramanujan Journal, 2(4):459-494, 1998.

Department of Mathematics, The University of North Carolina at Chapel Hill, CB \#3250, Phillips Hall, Chapel Hill, NC 27599

E-mail address: rimanyi@email.unc.edu

Department of MAThematics, University of Illinois at Urbana-Champaign, Urbana, IL 61801

E-mail address: weigndt2@uiuc.edu, ayong@uiuc.edu 